ESAIM: M2AN 48 (2014) 1061-1087

DOI: $10.1051 / \mathrm{m} 2 \mathrm{an} / 2014005$
ESAIM: Mathematical Modelling and Numerical Analysis

www.esaim-m2an.org

\title{
ANALYSIS OF A TIME DISCRETIZATION SCHEME FOR A NONSTANDARD VISCOUS CAHN-HILLIARD SYSTEM
}

\author{
Pierluigi Colli ${ }^{1}$, Gianni Gilardi ${ }^{1}$, Pavel Krejčíi ${ }^{2}$, Paolo Podio-Guidugli ${ }^{3}$ \\ AND JÜRGEN SPREKELS ${ }^{4}$
}

\begin{abstract}
In this paper we propose a time discretization of a system of two parabolic equations describing diffusion-driven atom rearrangement in crystalline matter. The equations express the balances of microforces and microenergy; the two phase fields are the order parameter and the chemical potential. The initial and boundary-value problem for the evolutionary system is known to be well posed. Convergence of the discrete scheme to the solution of the continuous problem is proved by a careful development of uniform estimates, by weak compactness and a suitable treatment of nonlinearities. Moreover, for the difference of discrete and continuous solutions we prove an error estimate of order one with respect to the time step.
\end{abstract}

Mathematics Subject Classification. 35A40, 35K55, 35Q70, 65M12, 65M15.

Received April 16, 2013. Revised November 5, 2013.

Published online June 30, 2014.

\section{INTRODUCTION}

This paper deals with a time discretization of a PDE system arising from a thermomechanical model for phase segregation by atom rearrangement on a lattice. The model was proposed by one of us in [29]; the resulting system has been analyzed in a recent and intensive research work by four of the present authors (see, in particular, $[16,17]$, both for well-posedness results and for a detailed presentation of the model).

Keywords and phrases. Cahn-Hilliard equation, phase field model, time discretization, convergence, error estimates.

1 Dipartimento di Matematica "F. Casorati", Università di Pavia, Via Ferrata 1, 27100 Pavia, Italy. pierluigi.colli@unipv.it; gianni.gilardi@unipv.it

2 Institute of Mathematics, Academy of Sciences of the Czech Republic, Zitna 25, 11567 Praha 1, Czech Republic.

krejci@math.cas.cz

3 Accademia Nazionale dei Lincei and Department of Mathematics, University of Rome TorVergata, Via della Ricerca Scientifica 1, 00133 Roma, Italy. ppg@uniroma2.it

4 Weierstraß-Institut für Angewandte Analysis und Stochastik, Mohrenstraße 39, 10117 Berlin, Germany.

sprekels@wias-berlin.de 
The initial and boundary value problem we consider consists in looking for two fields, the chemical potential $\mu>0$ and the order parameter $\rho \in(0,1)$, solving

$$
\begin{array}{cl}
(1+2 g(\rho)) \partial_{t} \mu+\mu \partial_{t} g(\rho)-\Delta \mu=0 & \text { in } \Omega \times(0, T), \\
\partial_{t} \rho-\Delta \rho+f^{\prime}(\rho)=\mu g^{\prime}(\rho) & \text { in } \Omega \times(0, T), \\
\partial_{\nu} \mu=\partial_{\nu} \rho=0 & \text { on } \Gamma \times(0, T), \\
\left.\mu\right|_{t=0}=\mu_{0} \text { and }\left.\rho\right|_{t=0}=\rho_{0} & \text { in } \Omega ;
\end{array}
$$

here $\Omega$ denotes a bounded domain of $\mathbb{R}^{3}$ with conveniently smooth boundary $\Gamma, T>0$ stands for some final time, and $\partial_{\nu}$ denotes differentiation in the direction of the outward normal $\nu$.

Problem (1.1)-(1.4) is parameterized by two nonlinear scalar-valued functions, $g$ and $f$, which enter into the definition of the system's free energy:

$$
\psi=\widehat{\psi}(\rho, \nabla \rho, \mu)=-\left(\frac{1}{2}+g(\rho)\right) \mu+f(\rho)+\frac{1}{2}|\nabla \rho|^{2} .
$$

We point out that in (1.1)-(1.5) all physical constants have been set equal to 1 . We also note that the last two terms in (1.5) favor phase segregation, the former because it introduces local energy minima, the latter because it penalizes spatial changes of the order parameter. For $g$, one can take any smooth function, provided it is nonnegative in the physically admissible domain:

$$
g(\rho) \geq 0 \text { for all } \rho \in(0,1)
$$

accordingly, the coefficient $1 / 2$ of $\mu$ in (1.5) should be regarded as a prescribed material bound. As to the possibly multi-well potential $f$, we take it to be the sum of two functions:

$$
f(\rho)=f_{1}(\rho)+f_{2}(\rho)
$$

the one, $f_{1}$, is convex over $(0,1)$, and such that its derivative $f_{1}^{\prime}$ (and possibly also $f_{1}$ ) is singular at the endpoints 0 and $1(c f .(2.3))$; the other is required to be smooth over the entire interval $[0,1]$, but not to have any convexity property, so that in equation (1.2) $f_{2}^{\prime}$ may serve as a non-monotone perturbation of the increasing function $f_{1}^{\prime}$.

As to the parameter functions, in [16] the choice made for $g$ was:

$$
g(\rho)=\rho,
$$

while the assumptions on $f$ were compatible with choosing a double-well potential:

$$
f(\rho)=\alpha_{1}\{\rho \ln (\rho)+(1-\rho) \ln (1-\rho)\}+\alpha_{2} \rho(1-\rho)+\alpha_{3} \rho,
$$

for some non-negative constants $\alpha_{1}, \alpha_{2}, \alpha_{3}$. Note that, if $\alpha_{3}$ is taken null, then, according to whether or not $2 \alpha_{1} \geq \alpha_{2}, f$ turns out to be convex in the whole of $[0,1]$ or it exhibits two wells, with a local maximum at $\rho=1 / 2$; moreover, for $\alpha_{3}>0$, the combined function:

$$
-g(\rho) \mu+f(\rho) \quad(\text { a part of } \psi)
$$

shows one global minimum in all cases, and it depends on the sign of $\left(\alpha_{3}-\mu\right)$ which minimum actually occurs. On the other hand, the framework of paper [17] allows for much more general choices of $g$ and $f$, as well as for nonlinear diffusion of $\mu$. Existence and uniqueness results were proved in both [16,17], with different approaches. Here, we take inspiration from arguments developed either in the one or in the other of those papers.

We introduce a time discretization of system (1.1)-(1.4) which is implicit with respect to the principal terms and tries to handle very carefully the nonlinearities. Namely, we address the recursive sequence of the elliptic 
problems:

$$
\begin{array}{cc}
\left(1+2 \gamma_{n}\right) \delta_{h} \mu_{n}+\mu_{n+1} \delta_{h} \gamma_{n}-\Delta \mu_{n+1}=0 & \text { in } \Omega, \\
\delta_{h} \rho_{n}-\Delta \rho_{n+1}+f^{\prime}\left(\rho_{n+1}\right)=\mu_{n} g^{\prime}\left(\rho_{n}\right) & \text { in } \Omega, \\
\partial_{\nu} \mu_{n+1}=\partial_{\nu} \rho_{n+1}=0 & \text { on } \Gamma,
\end{array}
$$

for $n=0,1, \ldots, N$, where $h=T / N$ is the time step, $\gamma_{n}:=g\left(\rho_{n}\right)$ and, for any $(N+1)$-ple $z_{0}, z_{1}, \ldots, z_{N}$, we let

$$
\delta_{h} z_{n}:=\left(z_{n+1}-z_{n}\right) / h \quad \text { for } n=0, \ldots, N-1 .
$$

After showing the existence of a discrete solution at any step, we carry out a number of uniform estimates on the time-discrete solution which allow us to prove convergence to the unique solution $(\mu, \rho)$ of the continuous problem (1.1)-(1.4), as $h$ tends to 0 (or, equivalently, $N$ goes to $+\infty$ ). Then, we estimate certain norms of the difference between the piecewise-linear-in-time interpolants of the discrete solutions and the continuous solution: more precisely, the first error estimate we prove is of order $h^{1 / 2}$; the second, which holds under stronger regularity assumptions on the initial data, is of order $h$.

We regard our results as a cornerstone in the construction of a time-and-space discretization of problem (1.1)-(1.4). With reference to such a complete discretization of Cahn-Hilliard and viscous CahnHilliard systems, we quote papers [1-8,21-23]. Some recent efforts can be found in the literature with the aim of analyzing other classes of phase transition problems, either to show existence via time discretization $[9,14,15,19,20,27,30,35,36]$ or to prove numerical results such as special convergence properties, stability or error estimates [11-13,18,25,28,31,33,34] ( $c f$. also [26] for a recent review on phase-field models). We dare say that our contribution goes deeply into the structure of the mathematical problem, because, as is not the case for many other similar investigations, we succeed in showing a linear order of convergence.

Our paper is organized as follows. In the next section, we list and discuss our assumptions, formulate the continuous and discrete problems precisely, and state our main results. Section 3 is devoted to proving that there is a discrete solution. The convergence result is proved in the long and articulate Section 4 . Finally, the last two Sections 5 and 6 contain detailed proofs of the two error estimates.

\section{MAin RESUlts}

In this section, we describe the mathematical problem under investigation, introduce the time discretization scheme, make our assumptions precise, and state our results.

First of all, we assume $\Omega$ to be a bounded connected open set in $\mathbb{R}^{3}$ with smooth boundary $\Gamma$. For convenience, we set

$$
V:=H^{1}(\Omega), \quad H:=L^{2}(\Omega), \quad \text { and } \quad W:=\left\{v \in H^{2}(\Omega): \partial_{\nu} v=0 \text { on } \Gamma\right\},
$$

and we endow these spaces with their standard norms, for which we use a self-explanatory notation like $\|\cdot\|_{V}$. The notation $\|\cdot\|_{p}(1 \leq p \leq+\infty)$ stands for the standard $L^{p}$-norm in $L^{p}(\Omega)$; for short, we sometimes do not distinguish between a space (or its norm) and a power thereof.

As to the parameter functions $f$ and $g$, we assume that

$$
\begin{aligned}
& f=f_{1}+f_{2}, \quad \text { where } \\
& f_{1}:(0,1) \rightarrow[0,+\infty) \text { is a convex } C^{2} \text { function satisfying } \\
& \qquad \lim _{r \searrow 0} f_{1}^{\prime}(r)=-\infty \text { and } \lim _{r \nearrow 1} f_{1}^{\prime}(r)=+\infty \\
& f_{2}:[0,1] \rightarrow \mathbb{R} \quad \text { is of class } C^{2} ; \\
& g:[0,1] \rightarrow \mathbb{R} \text { is of class } C^{2} \text { and nonnegative. }
\end{aligned}
$$


For the initial data, we require that

$$
\begin{aligned}
& \mu_{0} \in V \cap L^{\infty}(\Omega) \text { and } \quad \mu_{0} \geq 0 \text { a.e. in } \Omega ; \\
& \rho_{0} \in W \subset C^{0}(\bar{\Omega}) \text { and } \inf \rho_{0}>0, \sup \rho_{0}<1 .
\end{aligned}
$$

We stress that conditions (2.7) actually imply that $\rho_{0}$ is $1 / 2$-Hölder continuous: indeed, as $\Omega$ is a threedimensional domain, $W$ is continuosly embedded in $C^{0,1 / 2}(\bar{\Omega})$. As a consequence, also $f\left(\rho_{0}\right)$ and $f^{\prime}\left(\rho_{0}\right)$ are $1 / 2$-Hölder continuous, since $f$ and $f^{\prime}$ are smooth in $(0,1)$. On the other hand, we point out that in the sequel we will mostly exploit the compactness of the embedding $W \subset C^{0}(\bar{\Omega})$; Hölder continuity will play no role.

As recalled in the Introduction, in papers $[16,17]$ two versions of problem (1.1)-(1.4) were solved over an arbitrary time interval $[0, T]$ in a rather strong sense, because the solution pairs $(\mu, \rho)$ were required to satisfy

$$
\begin{aligned}
& \mu \in H^{1}(0, T ; H) \cap L^{2}(0, T ; W), \\
& \rho \in W^{1, \infty}(0, T ; H) \cap H^{1}(0, T ; V) \cap L^{\infty}(0, T ; W), \\
& \mu \geq 0 \quad \text { a.e. in } Q, \\
& 0<\rho<1 \quad \text { a.e. in } Q \text { and } \quad f^{\prime}(\rho) \in L^{\infty}(0, T ; H) .
\end{aligned}
$$

Note that the boundary conditions (1.3) follow from (2.8)-(2.9), due to the definition of $W$ in (2.1). Accordingly, the solutions to the problems of type (1.1)-(1.4) studied in $[16,17]$ were pairs $(\mu, \rho)$ satisfying, in addition to $(2.8)-(2.11)$, the system

$$
\begin{array}{cc}
(1+2 g(\rho)) \partial_{t} \mu+\mu \partial_{t} g(\rho)-\Delta \mu=0 & \text { a.e. in } Q, \\
\partial_{t} \rho-\Delta \rho+f^{\prime}(\rho)=\mu g^{\prime}(\rho) & \text { a.e. in } Q, \\
\mu(0)=\mu_{0} \quad \text { and } \quad \rho(0)=\rho_{0} & \text { a.e. in } \Omega .
\end{array}
$$

Some of the results proved in the quoted papers are summarized in the following theorem.

Theorem 2.1. Let assumptions (2.2)-(2.7) hold. Then, there exists a unique pair $(\mu, \rho)$ satisfying $(2.8)-(2.11)$ and solving problem (2.12)-(2.14). Moreover, $\mu \in L^{\infty}(Q)$, and there exist $\rho_{*}, \rho^{*} \in(0,1)$ such that $\rho_{*} \leq \rho \leq \rho^{*}$ a.e. in $Q$.

The main aim of the present paper is to show that, given the time-discretization scheme introduced here below, the discrete solution converges to the solution $(\mu, \rho)$ as the time step $h$ tends to zero.

Notation 2.2. Assume that $N$ is a positive integer, and let $Z$ be any normed space. We define $\delta_{h}: Z^{N+1} \rightarrow Z^{N}$ as follows:

$$
\begin{aligned}
& \text { for } z=\left(z_{0}, z_{1}, \ldots, z_{N}\right) \in Z^{N+1} \text { and } w=\left(w_{0}, \ldots, w_{N-1}\right) \in Z^{N}, \\
& \delta_{h} z_{n}=\left(w_{n}\right) \text { means that } w_{n}=\frac{z_{n+1}-z_{n}}{h} \text { for } n=0, \ldots, N-1 .
\end{aligned}
$$

We can also iterate the discretization procedure, and define, e.g.,

$$
\delta_{h}^{2} z_{n}:=\frac{\delta_{h} z_{n+1}-\delta_{h} z_{n}}{h}=\frac{z_{n+2}-2 z_{n+1}+z_{n}}{h^{2}} \text { for } n=0, \ldots, N-2 .
$$

Next, by setting $h:=T / N$ (without stressing the dependence of $h$ on $N)$ and $I_{n}:=((n-1) h, n h)$ for $n=$ $1, \ldots, N$, we introduce the interpolation maps from $Z^{N+1}$ into either $L^{\infty}(0, T ; Z)$ or $W^{1, \infty}(0, T ; Z)$ as follows: for $z=\left(z_{0}, z_{1}, \ldots, z_{N}\right) \in Z^{N+1}$, we set

$$
\begin{aligned}
& \bar{z}_{h}, \underline{z}_{h} \in L^{\infty}(0, T ; Z) \quad \text { and } \quad \widehat{z}_{h} \in W^{1, \infty}(0, T ; Z), \\
& \bar{z}_{h}(t)=z_{n} \quad \text { and } \quad \underline{z}_{h}(t)=z_{n-1} \quad \text { for a.a. } t \in I_{n}, n=1, \ldots, N, \\
& \widehat{z}_{h}(0)=z_{0} \quad \text { and } \quad \partial_{t} \widehat{z}_{h}(t)=\delta_{h} z_{n-1} \quad \text { for a.a. } t \in I_{n}, n=1, \ldots, N .
\end{aligned}
$$


These maps yield the backward/forward piecewise-constant and piecewise-linear interpolants of the discrete vectors. We obviously have:

$$
\begin{aligned}
& \left\|\bar{z}_{h}\right\|_{L^{\infty}(0, T ; Z)}=\max _{n=1, \ldots, N}\left\|z_{n}\right\|_{Z}, \quad\left\|\underline{z}_{h}\right\|_{L^{\infty}(0, T ; Z)}=\max _{n=0, \ldots, N-1}\left\|z_{n}\right\|_{Z}, \\
& \left\|\bar{z}_{h}\right\|_{L^{2}(0, T ; Z)}^{2}=h \sum_{n=1}^{N}\left\|z_{n}\right\|_{Z}^{2}, \quad\left\|\underline{z}_{h}\right\|_{L^{2}(0, T ; Z)}^{2}=h \sum_{n=0}^{N-1}\left\|z_{n}\right\|_{Z}^{2} .
\end{aligned}
$$

Moreover, as $\widehat{z}_{h}(t)$ is a convex combination of $z_{n-1}$ and $z_{n}$ for $t \in I_{n}$, we also have

$$
\begin{aligned}
& \left\|\widehat{z}_{h}\right\|_{L^{\infty}(0, T ; Z)}=\max _{n=0, \ldots, N}\left\|z_{n}\right\|_{Z}=\max \left\{\left\|z_{0}\right\|_{Z},\left\|\bar{z}_{h}\right\|_{L^{\infty}(0, T ; Z)}\right\}, \\
& \left\|\widehat{z}_{h}\right\|_{L^{2}(0, T ; Z)}^{2} \leq h \sum_{n=1}^{N}\left(\left\|z_{n-1}\right\|_{Z}^{2}+\left\|z_{n}\right\|_{Z}^{2}\right) \leq h\left\|z_{0}\right\|_{Z}^{2}+2\left\|\bar{z}_{h}\right\|_{L^{2}(0, T ; Z)}^{2} .
\end{aligned}
$$

Finally, by a direct computation, it is straightforward to prove that

$$
\begin{gathered}
\left\|\bar{z}_{h}-\widehat{z}_{h}\right\|_{L^{\infty}(0, T ; Z)}=\max _{n=0, \ldots, N-1}\left\|z_{n+1}-z_{n}\right\|_{Z}=h\left\|\partial_{t} \widehat{z}_{h}\right\|_{L^{\infty}(0, T ; Z)}, \\
\left\|\bar{z}_{h}-\widehat{z}_{h}\right\|_{L^{2}(0, T ; Z)}^{2}=\frac{h}{3} \sum_{n=0}^{N-1}\left\|z_{n+1}-z_{n}\right\|_{Z}^{2}=\frac{h^{2}}{3}\left\|\partial_{t} \widehat{z}_{h}\right\|_{L^{2}(0, T ; Z)}^{2},
\end{gathered}
$$

and that the same identities hold for the difference $\underline{z}_{h}-\widehat{z}_{h}$.

At this point, we can write the discrete scheme presented in the Introduction in a precise form. For any positive integer $N$, we look for two vectors $\left(\mu_{n}\right)_{n=0}^{N}$ and $\left(\rho_{n}\right)_{n=0}^{N}$ satisfying the following conditions:

i) the first components $\mu_{0}$ and $\rho_{0}$ coincide with the initial data;

ii) for $n=0, \ldots, N-1$, we have that

$$
\mu_{n+1}, \rho_{n+1} \in W, \quad \mu_{n+1} \geq 0 \quad \text { and } \quad 0<\rho_{n+1}<1 \quad \text { in } \Omega, \quad f^{\prime}\left(\rho_{n+1}\right) \in H ;
$$

iii) if $\left(\gamma_{n}\right)_{n=0}^{N}$ is the vector whose components are $\gamma_{n}:=g\left(\rho_{n}\right)$, there hold

$$
\begin{aligned}
& \left(1+2 \gamma_{n}\right) \delta_{h} \mu_{n}+\mu_{n+1} \delta_{h} \gamma_{n}-\Delta \mu_{n+1}=0, \\
& \delta_{h} \rho_{n}-\Delta \rho_{n+1}+f^{\prime}\left(\rho_{n+1}\right)=\mu_{n} g^{\prime}\left(\rho_{n}\right),
\end{aligned}
$$

for $n=0, \ldots, N-1$.

Also in this case, the homogenous Neumann boundary conditions are implicit in the regularity requirements (see $(2.26)$ and (2.1)).

Clearly, the "true" problem consists in finding $\left(\mu_{n+1}, \rho_{n+1}\right)$ once $\left(\rho_{n}, \mu_{n}\right)$ is given. Here is our result in this direction.

Theorem 2.3. Assume (2.2)-(2.7). Then, there exists $h_{0}>0$ such that, for $h<h_{0}$ and $n=0, \ldots, N-1$, problem $(2.27)-(2.28)$ has a unique solution $\left(\mu_{n+1}, \rho_{n+1}\right)$ satisfying $(2.26)$.

Our next results concern firstly convergence of interpolants for vectors $\left(\rho_{n}\right)$ and $\left(\mu_{n}\right)$ to the solution $(\mu, \rho)$ to problem (2.12)-(2.14), then error estimates. We point out that, for simplicity, the convergence theorem here below is not stated in a precise form: the topological setting will be specified later, by means of relations (4.34)-(4.38).

Theorem 2.4. Assume (2.2)-(2.7). Then, in accord with Notation 2.2, the sequences of interpolants for the discrete solutions given by Theorem 2.3 converge to the solution $(\mu, \rho)$ given by Theorem 2.1 as $h$ tends to 0 , in a suitable topology. 
Theorem 2.5. In addition to (2.2)-(2.7), assume that

$$
\rho_{0} \in H^{3}(\Omega) .
$$

Then, for sufficiently small $h>0$, the following error estimate holds:

$$
\left\|\widehat{\rho}_{h}-\rho\right\|_{H^{1}(0, T ; H) \cap L^{\infty}(0, T ; V)}+\left\|\widehat{\mu}_{h}-\mu\right\|_{L^{\infty}(0, T ; H) \cap L^{2}(0, T ; V)} \leq c h^{1 / 2},
$$

where $c$ depends only on the structural assumptions and the data.

Theorem 2.6. In addition to (2.2)-(2.7), assume (2.29) and

$$
\mu_{0} \in W \text {. }
$$

Then, for sufficiently small $h>0$, the following error estimate holds:

$$
\left\|\widehat{\rho}_{h}-\rho\right\|_{H^{1}(0, T ; H) \cap L^{\infty}(0, T ; V)}+\left\|\widehat{\mu}_{h}-\mu\right\|_{L^{\infty}(0, T ; H) \cap L^{2}(0, T ; V)} \leq c h,
$$

where $c$ depends only on the structural assumptions and the data.

Remark 2.7. It is easy to see that our assumptions (2.2)-(2.7) ensure that both $f^{\prime}\left(\rho_{0}\right)$ and $\mu_{0} g^{\prime}\left(\rho_{0}\right)$ belong to $V$. It follows that $(2.29)$ is equivalent to

$$
-\Delta \rho_{0}+f^{\prime}\left(\rho_{0}\right)-\mu_{0} g^{\prime}\left(\rho_{0}\right) \in V .
$$

We also notice that the assumptions (2.29) and (2.31) ensure further regularity for the solution $(\mu, \rho)$ to the continuous problem (see the forthcoming Rem. 6.1).

We prove Theorem 2.3 in Section 3 and Theorem 2.4 in Section 4; the last two sections are devoted to proving, respectively, Theorems 2.5 and 2.6 .

Throughout the paper, we account for the well-known embeddings $V \subset L^{q}(\Omega)(1 \leq q \leq 6)$ and $W \subset C^{0}(\bar{\Omega})$, and for the related Sobolev inequalities:

$$
\|v\|_{q} \leq C\|v\|_{V} \text { and }\|v\|_{\infty} \leq C\|v\|_{W},
$$

for $v \in V$ and $v \in W$, respectively, where $C$ depends on $\Omega$ only, since sharpness is not needed. We remark that these embeddings are compact provided that $q<6$. In particular, the following compactness inequality holds:

$$
\|v\|_{4} \leq \sigma\|\nabla v\|_{H}+C_{\sigma}\|v\|_{H}, \quad \text { for every } v \in V \text { and } \sigma>0
$$

in (2.35), $C_{\sigma}$ is a constant that depends only on $\Omega$ and $\sigma$. Furthermore, we make repeated use of Hölder's inequality, of the following elementary identity:

$$
(a-b) a=\frac{1}{2} a^{2}-\frac{1}{2} b^{2}+\frac{1}{2}(a-b)^{2}, \quad \text { for every } a, b \in \mathbb{R}
$$

and of Young's inequality

$$
a b \leq \sigma a^{2}+\frac{1}{4 \sigma} b^{2}, \quad \text { for every } a, b \geq 0 \text { and } \sigma>0 .
$$

Moreover, we use the discrete Gronwall lemma in the following form (see, e.g., [24], Prop. 2.2.1): if $\left(a_{0}, \ldots, a_{N}\right) \in$ $[0,+\infty)^{N+1}$ and $\left(b_{1}, \ldots, b_{N}\right) \in[0,+\infty)^{N}$ satisfy

$$
\begin{aligned}
& a_{m} \leq a_{0}+\sum_{n=1}^{m-1} b_{n} a_{n} \quad \text { for } m=1, \ldots, N, \text { then } \\
& a_{m} \leq a_{0} \exp \left(\sum_{n=1}^{m-1} b_{n}\right) \quad \text { for } m=1, \ldots, N .
\end{aligned}
$$


Finally, throughout the paper we use a small-case italic $c$ for a number of different constants that may only depend on $\Omega$, the final time $T$, the shape of $f$, the properties of the data involved in the statements at hand; those constants we need to refer to are always denoted by capital letters, just like $C$ in (2.34). Moreover, a notation like $c_{\sigma}$ signals a constant that depends also on the parameter $\sigma$. The reader should keep in mind that the meaning of $c$ and $c_{\sigma}$ might change from line to line and even in the same chain of inequalities and that their values never depend on the time step $h$.

\section{ExistenCE}

In this section, we prove Theorem 2.3. We argue inductively with respect to $n$, i.e., by assuming that a pair $\left(\mu_{n}, \rho_{n}\right)$ satisfying (2.26) with $n$ in place of $n+1$ is given, we prove that problem (2.27)-(2.28) has a unique solution $\left(\mu_{n+1}, \rho_{n+1}\right)$ satisfying (2.26). More precisely, as is going to be clear from the proof, we need less regularity for $\mu_{n}$, e.g., $\mu_{n} \in V$. In particular, our assumptions on $\mu_{0}$ are sufficient to start. We rewrite (2.27)-(2.28) in the form

$$
\begin{aligned}
& \left(1+\gamma_{n}+\gamma_{n+1}\right) \mu_{n+1}-h \Delta \mu_{n+1}=\left(1+2 \gamma_{n}\right) \mu_{n}, \\
& \rho_{n+1}-h \Delta \rho_{n+1}+h f^{\prime}\left(\rho_{n+1}\right)=\rho_{n}+h \mu_{n} g^{\prime}\left(\rho_{n}\right),
\end{aligned}
$$

and solve (3.2) first for $\rho_{n+1}$ (so that $\gamma_{n+1}$ is also known), then (3.1). In order to solve both problems, it is expedient to replace each equation by a minimum problem, at least for $h$ small enough. We consider the functionals:

$$
\begin{aligned}
& J_{1}: V \rightarrow \mathbb{R} \text { and } J_{2}: V \rightarrow(-\infty,+\infty], \quad \text { defined by, respectively, } \\
& J_{1}(v):=\frac{h}{2} \int_{\Omega}|\nabla v|^{2}+\frac{1}{2} \int_{\Omega}\left(1+\gamma_{n}+\gamma_{n+1}\right) v^{2}-\int_{\Omega}\left(1+2 \gamma_{n}\right) \mu_{n} v \text { and } \\
& J_{2}(v):=\frac{h}{2} \int_{\Omega}|\nabla v|^{2}+\frac{1}{2} \int_{\Omega} v^{2}+h \int_{\Omega} \tilde{f}(v)-\int_{\Omega}\left(\rho_{n}+h \mu_{n} g^{\prime}\left(\rho_{n}\right)\right) v .
\end{aligned}
$$

In (3.4), we have $\tilde{f}=\tilde{f}_{1}+\tilde{f}_{2}$, where $\tilde{f}_{2}$ is any smooth extension of $f_{2}$ to the whole of $\mathbb{R}$ and $\tilde{f}_{1}$ is the unique convex and lower semicontinuous extension of $f_{1}$ that satisfies $\tilde{f}(r)=+\infty$ if $r \notin[0,1]$. By the way, it is understood that the corresponding integral that appears in (3.4) is infinite if $\tilde{f}(v)$ does not belong to $L^{1}(\Omega)$. Therefore, both functionals are well-defined and proper whenever $\mu_{n} \in V$ and $\rho_{n} \in W$ (and this implies boundedness of $g\left(\rho_{n}\right)$ and $\left.g^{\prime}\left(\rho_{n}\right)\right)$. Moreover, in view of the above remarks, $J_{1}$ is continuous, and $J_{2}$ lower semicontinuous, on $V$.

Now, we observe that equations (3.1) and (3.2), when complemented by the regularity requirements in (2.26) (which yield the homogeneous Neumann boundary conditions), are the strong forms of the Euler-Lagrange variational equations for the stationary points of $J_{1}$ and $J_{2}$, respectively. More precisely, the strong form (3.1) follows from the variational formulation thanks to the regularity theory of elliptic equations. As far as (3.2) is concerned, the function $f^{\prime}$ should be replaced - in principle, at least - by the sum $\partial \tilde{f}_{1}+\tilde{f}_{2}^{\prime}$, where $\partial \tilde{f}_{1}$ is the subdifferential of $\tilde{f}_{1}$. However, once an $L^{2}(\Omega)$-estimate is obtained for the subdifferential (and standard arguments of the theory of maximal monotone operators easily yield such an estimate, see, e.g., [10]), the variational Euler-Lagrange equation can be written exactly in the form (3.2), because $\partial \tilde{f}_{1}$ is single-valued due to our assumptions on $f_{1}$ (see (2.3), in particular). Consequently, existence and uniqueness of the solution $\left(\mu_{n}, \rho_{n}\right)$ follow if the functionals (3.3) and (3.4) are convex, so that each of the correponding minimum problems has a unique solution. This is granted for the first problem: indeed, $J_{1}$ is strictly convex and coercive, because $g$ is nonnegative. The same holds for $J_{2}$, provided that the second derivative of function $r \mapsto r^{2} / 2+h f_{2}(r)$ is strictly positive on $[0,1]$, which is the case if $h \sup \left|f_{2}^{\prime \prime}\right|<1$.

It remains to prove that $\mu_{n+1} \geq 0$. To this end, we multiply (3.1) by $-\mu_{n+1}^{-}$, where $v^{-}=\max \{-v, 0\}$ denotes the negative part of $v$, and integrate over $\Omega$. We obtain:

$$
\int_{\Omega}\left(1+g\left(\rho_{n}\right)+g\left(\rho_{n+1}\right)\right)\left|\mu_{n+1}^{-}\right|^{2}+h \int_{\Omega}\left|\nabla \mu_{n+1}^{-}\right|^{2}=-\int_{\Omega}\left(1+2 g\left(\rho_{n}\right)\right) \mu_{n} \mu_{n+1}^{-} \leq 0,
$$

because both $g$ and $\mu_{n}$ are nonnegative. This implies that $\mu_{n+1}^{-}=0$, and hence that $\mu_{n+1} \geq 0$. 


\section{Convergence}

In this section, we prove Theorem 2.4. For convenience, we introduce one more vector, $\left(\xi_{n}\right)_{n=0}^{N}$, and recall the definition of $\left(\gamma_{n}\right)_{n=0}^{N}$ :

$$
\xi_{n}:=f_{1}^{\prime}\left(\rho_{n}\right) \quad \text { and } \quad \gamma_{n}:=g\left(\rho_{n}\right) \text { for } n=0, \ldots, N
$$

Later on, we also use the interpolants of these vectors according to Notation 2.2. Our argument uses compactness and monotonicity methods.

First a priori estimate. We multiply (3.1) by $\mu_{n+1}$ and integrate over $\Omega$. By accounting for the elementary identity (2.36), we obtain

$$
\begin{aligned}
& \frac{1}{2} \int_{\Omega} \mu_{n+1}^{2}-\frac{1}{2} \int_{\Omega} \mu_{n}^{2}+\frac{1}{2} \int_{\Omega}\left|\mu_{n+1}-\mu_{n}\right|^{2}+h \int_{\Omega}\left|\nabla \mu_{n+1}\right|^{2} \\
& +\int_{\Omega}\left(\gamma_{n} \mu_{n+1}^{2}+\gamma_{n+1} \mu_{n+1}^{2}-2 \gamma_{n} \mu_{n} \mu_{n+1}\right)=0 .
\end{aligned}
$$

As $\gamma_{n} \mu_{n+1}^{2}+\gamma_{n+1} \mu_{n+1}^{2}-2 \gamma_{n} \mu_{n} \mu_{n+1}=\gamma_{n+1} \mu_{n+1}^{2}-\gamma_{n} \mu_{n}^{2}+\gamma_{n}\left(\mu_{n+1}-\mu_{n}\right)^{2}$, we derive that

$$
\begin{aligned}
& \int_{\Omega}\left(\frac{1}{2}+\gamma_{n+1}\right) \mu_{n+1}^{2}-\int_{\Omega}\left(\frac{1}{2}+\gamma_{n}\right) \mu_{n}^{2}+\int_{\Omega}\left(\frac{1}{2}+\gamma_{n}\right)\left|\mu_{n+1}-\mu_{n}\right|^{2} \\
& \quad+h \int_{\Omega}\left|\nabla \mu_{n+1}\right|^{2}=0 .
\end{aligned}
$$

On summing over $n=0, \ldots, m-1$ with $1 \leq m \leq N$, we conclude that

$$
\begin{aligned}
& \int_{\Omega}\left(\frac{1}{2}+\gamma_{m}\right) \mu_{m}^{2}+h^{2} \sum_{n=0}^{m-1} \int_{\Omega}\left(\frac{1}{2}+\gamma_{n}\right)\left|\delta_{h} \mu_{n}\right|^{2}+h \sum_{n=0}^{m-1} \int_{\Omega}\left|\nabla \mu_{n+1}\right|^{2} \\
& =\int_{\Omega}\left(\frac{1}{2}+\gamma_{0}\right) \mu_{0}^{2} \quad \text { for } m=1, \ldots, N .
\end{aligned}
$$

As $g$ is nonnegative and hence $\gamma_{i} \geq 0$, this implies that $\left\|\mu_{m}\right\|_{H} \leq c$ for $m=1, \ldots, N$. Thus, the above estimate also yields

$$
\max _{m=1, \ldots, N}\left\|\mu_{m}\right\|_{H}^{2}+h^{2} \sum_{n=0}^{N-1}\left\|\delta_{h} \mu_{n}\right\|_{H}^{2}+h \sum_{n=1}^{N}\left\|\mu_{n}\right\|_{V}^{2} \leq c .
$$

In terms of the interpolants introduced in Notation 2.2, with the help of $\mu_{0} \in V,(2.20)-(2.21)$, and (2.24)-(2.25) we have that

$$
\begin{aligned}
& \left\|\bar{\mu}_{h}\right\|_{L^{\infty}(0, T ; H) \cap L^{2}(0, T ; V)}^{2}+\left\|\underline{\mu}_{h}\right\|_{L^{\infty}(0, T ; H) \cap L^{2}(0, T ; V)}^{2} \\
& \quad+\left\|\widehat{\mu}_{h}\right\|_{L^{\infty}(0, T ; H) \cap L^{2}(0, T ; V)}^{2}+h\left\|\partial_{t} \widehat{\mu}_{h}\right\|_{L^{2}(0, T ; H)}^{2} \leq c .
\end{aligned}
$$

Second $\boldsymbol{a}$ priori estimate. In (3.2), we move $\rho_{n}$ to the left-hand side. Then, we multiply by $\rho_{n+1}-\rho_{n}$ and integrate over $\Omega$. We obtain

$$
\begin{aligned}
& \int_{\Omega}\left|\rho_{n+1}-\rho_{n}\right|^{2}+\frac{h}{2} \int_{\Omega}\left|\nabla \rho_{n+1}\right|^{2}-\frac{h}{2} \int_{\Omega}\left|\nabla \rho_{n}\right|^{2}+\frac{h}{2} \int_{\Omega}\left|\nabla \rho_{n+1}-\nabla \rho_{n}\right|^{2} \\
& +h \int_{\Omega} f^{\prime}\left(\rho_{n+1}\right)\left(\rho_{n+1}-\rho_{n}\right)=h \int_{\Omega} \mu_{n} g^{\prime}\left(\rho_{n}\right)\left(\rho_{n+1}-\rho_{n}\right) .
\end{aligned}
$$


Now, we consider the last integral on the left-hand side of (4.4). We split $f^{\prime}=f_{1}^{\prime}+f_{2}^{\prime}$ and use the convexity assumption of $f_{1}$ and boundedness for $f_{2}^{\prime}$. We get

$$
\int_{\Omega} f^{\prime}\left(\rho_{n+1}\right)\left(\rho_{n+1}-\rho_{n}\right) \geq \int_{\Omega}\left(f_{1}\left(\rho_{n+1}\right)-f_{1}\left(\rho_{n}\right)\right)-c \int_{\Omega}\left|\rho_{n+1}-\rho_{n}\right| .
$$

Since also $g^{\prime}$ is bounded, we infer from (4.4) that

$$
\begin{aligned}
& \int_{\Omega}\left|\rho_{n+1}-\rho_{n}\right|^{2}+\frac{h}{2} \int_{\Omega}\left|\nabla \rho_{n+1}\right|^{2}-\frac{h}{2} \int_{\Omega}\left|\nabla \rho_{n}\right|^{2}+\frac{h}{2} \int_{\Omega}\left|\nabla \rho_{n+1}-\nabla \rho_{n}\right|^{2} \\
& \quad+h \int_{\Omega}\left(f_{1}\left(\rho_{n+1}\right)-f_{1}\left(\rho_{n}\right)\right) \leq c h \int_{\Omega}\left(1+\mu_{n}\right)\left|\rho_{n+1}-\rho_{n}\right| \\
& \leq \frac{1}{2} \int_{\Omega}\left|\rho_{n+1}-\rho_{n}\right|^{2}+c h^{2} \int_{\Omega}\left(1+\mu_{n}^{2}\right) \leq \frac{1}{2} \int_{\Omega}\left|\rho_{n+1}-\rho_{n}\right|^{2}+c h^{2},
\end{aligned}
$$

the last inequality being due to (4.3). By dividing by $h$, summing over $n=0, \ldots, m-1$, and owing to the obvious inequality $m h \leq c$, we conclude that

$$
h \sum_{n=0}^{m-1} \int_{\Omega}\left|\delta_{h} \rho_{n}\right|^{2}+\frac{1}{2} \int_{\Omega}\left|\nabla \rho_{m+1}\right|^{2}+h^{2} \sum_{n=0}^{m-1} \int_{\Omega}\left|\delta_{h} \nabla \rho_{n}\right|^{2}+\int_{\Omega} f_{1}\left(\rho_{m+1}\right) \leq c
$$

for $m=0, \ldots, N-1$. As the term involving the difference quotient $\delta_{h} \rho_{N-1}$ is missing in the first sum since $m \leq N-1$, we estimate it directly. We multiply (2.28), written for $n=N-1$, by $h \delta_{h} \rho_{N-1}$ and integrate over $\Omega$. We have

$$
h \int_{\Omega}\left|\delta_{h} \rho_{N-1}\right|^{2}+\int_{\Omega}\left(\nabla \rho_{N}-\nabla \rho_{N-1}\right) \cdot \nabla \rho_{N}+\int_{\Omega} f_{1}^{\prime}\left(\rho_{N}\right)\left(\rho_{N}-\rho_{N-1}\right)=h \int_{\Omega} \phi \delta_{h} \rho_{N-1},
$$

where we have set $\phi:=\mu_{N-1} g^{\prime}\left(\rho_{N-1}\right)-f_{2}^{\prime}\left(\rho_{N-1}\right)$. Owing to the elementary identity $(2.36)$ and to the convexity of $f_{1}$ as before, we have

$$
\begin{aligned}
& h\left\|\delta_{h} \rho_{N-1}\right\|_{H}^{2}+\frac{1}{2}\left\|\nabla \rho_{N}\right\|_{H}^{2}+\frac{1}{2}\left\|\nabla \rho_{N}-\nabla \rho_{N-1}\right\|_{H}^{2}+\int_{\Omega} f_{1}\left(\rho_{N}\right) \\
& \leq \frac{1}{2}\left\|\nabla \rho_{N-1}\right\|_{H}^{2}+\int_{\Omega} f_{1}\left(\rho_{N-1}\right)+h\|\phi\|_{H}\left\|\delta_{h} \rho_{N-1}\right\|_{H} \\
& \leq \frac{1}{2}\left\|\nabla \rho_{N-1}\right\|_{H}^{2}+\int_{\Omega} f_{1}\left(\rho_{N-1}\right)+\frac{h}{2}\|\phi\|_{H}^{2}+\frac{h}{2}\left\|\delta_{h} \rho_{N-1}\right\|_{H} .
\end{aligned}
$$

Now, we observe that the first two terms of the last line are bounded by (4.5) written with $m=N-2$ and that $\phi$ is estimated in $H$ thanks to (4.2) and our assumptions of $g$ and $f_{2}$. Moreover, the last term of the first line can be ignored since $f_{1}$ is nonnegative. Hence, we get the desired bound for the first term. At this point, we can easily derive an estimate for $\left\|\rho_{m}\right\|_{H}$ for $m=1, \ldots, N$. By using the obvious identity $\rho_{m}=\rho_{0}+h \sum_{n=0}^{m-1} \delta_{h} \rho_{n}$ and the Euclidean Schwarz and Young inequalities, we see that

$$
\left\|\rho_{m}\right\|_{H} \leq\left\|\rho_{0}\right\|_{H}+h \sum_{n=0}^{m-1}\left\|\delta_{h} \rho_{n}\right\|_{H} \leq c+\frac{h}{2}\left(m+\sum_{n=0}^{m-1}\left\|\delta_{h} \rho_{n}\right\|_{H}^{2}\right) \leq c .
$$

Hence, by recalling (4.5) and our last estimates, we conclude that

$$
h \sum_{n=0}^{N-1}\left\|\delta_{h} \rho_{n}\right\|_{H}^{2}+\max _{m=1, \ldots, N}\left\|\rho_{m}\right\|_{V}^{2}+h^{2} \sum_{n=0}^{N-2}\left\|\delta_{h} \nabla \rho_{n}\right\|_{H}^{2} \leq c .
$$


In terms of the interpolants, (4.6) reads (thanks also to $\rho_{0} \in V$ and to (2.20)-(2.21) and (2.24))

$$
\begin{aligned}
\left\|\partial_{t} \widehat{\rho}_{h}\right\|_{L^{2}(0, T ; H)}^{2}+\left\|\bar{\rho}_{h}\right\|_{L^{\infty}(0, T ; V)}^{2}+\left\|\underline{\rho}_{h}\right\|_{L^{\infty}(0, T ; V)}^{2} & \\
& +\left\|\widehat{\rho}_{h}\right\|_{L^{\infty}(0, T ; V)}^{2}+h\left\|\partial_{t} \nabla \widehat{\rho}_{h}\right\|_{L^{2}(0, T-h ; H)}^{2} \leq c .
\end{aligned}
$$

Third a priori estimate. We come back to (2.28) and rewrite it as (recall (4.1))

$$
-\Delta \rho_{n+1}+\xi_{n+1}=-\delta_{h} \rho_{n}+\mu_{n} g^{\prime}\left(\rho_{n}\right)-f_{2}^{\prime}\left(\rho_{n+1}\right) .
$$

Hence, a standard argument (multiplying by $-\Delta \rho_{n+1}$ and by $\xi_{n+1}$ ) shows that the following estimate holds true

$$
\left\|\Delta \rho_{n+1}\right\|_{H}+\left\|\xi_{n+1}\right\|_{H} \leq c\left\|-\delta_{h} \rho_{n}+\mu_{n} g^{\prime}\left(\rho_{n}\right)-f_{2}^{\prime}\left(\rho_{n+1}\right)\right\|_{H}
$$

Thus, we infer that

$$
\left\|\Delta \rho_{n+1}\right\|_{H}^{2}+\left\|\xi_{n+1}\right\|_{H}^{2} \leq c\left(\left\|\delta_{h} \rho_{n}\right\|_{H}^{2}+\left\|\mu_{n}\right\|_{H}^{2}+1\right) \quad \text { for } n=0, \ldots, N-1 .
$$

Moreover, by using the regularity theory of elliptic equations, we deduce that

$$
\left\|\rho_{n+1}\right\|_{W}^{2}+\left\|\xi_{n+1}\right\|_{H}^{2} \leq c\left(\left\|\rho_{n+1}\right\|_{V}^{2}+\left\|\delta_{h} \rho_{n}\right\|_{H}^{2}+\left\|\mu_{n}\right\|_{H}^{2}+1\right) .
$$

Now, we multiply (4.8) by $h$ and sum over $n=0, \ldots, m-1$. By accounting for (4.2) and (4.6), we conclude that

$$
h \sum_{n=0}^{N-1}\left\|\rho_{n+1}\right\|_{W}^{2}+h \sum_{n=0}^{N-1}\left\|\xi_{n+1}\right\|_{H}^{2} \leq c .
$$

In terms of the interpolants, (4.10) yields (by accounting for $\rho_{0} \in W$ )

$$
\left\|\bar{\rho}_{h}\right\|_{L^{2}(0, T ; W)}^{2}+\left\|\underline{\rho}_{h}\right\|_{L^{2}(0, T ; W)}^{2}+\left\|\widehat{\rho}_{h}\right\|_{L^{2}(0, T ; W)}^{2} \leq c
$$

besides an estimate for, e.g., $\left\|\bar{\xi}_{h}\right\|$ in $L^{2}(0, T ; H)$.

Fourth $\boldsymbol{a}$ priori estimate. We write (2.28) with $n+1$ in place of $n$ and take the difference between the obtained equality and (2.28) itself. Then we multiply this difference by $\delta_{h} \rho_{n+1}$ and integrate over $\Omega$. We have

$$
\begin{aligned}
& \int_{\Omega}\left(\delta_{h} \rho_{n+1}-\delta_{h} \rho_{n}\right) \delta_{h} \rho_{n+1}+\int_{\Omega}\left(\nabla \rho_{n+2}-\nabla \rho_{n+1}\right) \cdot \delta_{h} \nabla \rho_{n+1} \\
& \quad+\int_{\Omega}\left(f^{\prime}\left(\rho_{n+2}\right)-f^{\prime}\left(\rho_{n+1}\right)\right) \delta_{h} \rho_{n+1}=\int_{\Omega}\left(\mu_{n+1} g^{\prime}\left(\rho_{n+1}\right)-\mu_{n} g^{\prime}\left(\rho_{n}\right)\right) \delta_{h} \rho_{n+1} .
\end{aligned}
$$

By accounting for the elementary identity (2.36), we get

$$
\int_{\Omega}\left(\delta_{h} \rho_{n+1}-\delta_{h} \rho_{n}\right) \delta_{h} \rho_{n+1}=\frac{1}{2} \int_{\Omega}\left|\delta_{h} \rho_{n+1}\right|^{2}-\frac{1}{2} \int_{\Omega}\left|\delta_{h} \rho_{n}\right|^{2}+\frac{1}{2} \int_{\Omega}\left|\delta_{h} \rho_{n+1}-\delta_{h} \rho_{n}\right|^{2} .
$$

Moreover, the second integral on the left-hand side of (4.12) can be written in terms of $\delta_{h} \rho_{n+1}$ in an obvious way. Finally, by splitting $f^{\prime}$ into $f_{1}^{\prime}+f_{2}^{\prime}$, observing that the contribution due to the terms involving $f_{1}^{\prime}$ is nonnegative since $f_{1}^{\prime}$ is monotone and moving the other ones to the right-hand side, we see that (4.12) yields the inequality

$$
\begin{aligned}
& \frac{1}{2} \int_{\Omega}\left|\delta_{h} \rho_{n+1}\right|^{2}-\frac{1}{2} \int_{\Omega}\left|\delta_{h} \rho_{n}\right|^{2}+\frac{1}{2} \int_{\Omega}\left|\delta_{h} \rho_{n+1}-\delta_{h} \rho_{n}\right|^{2}+h \int_{\Omega}\left|\delta_{h} \nabla \rho_{n+1}\right|^{2} \\
& \leq-\int_{\Omega}\left(f_{2}^{\prime}\left(\rho_{n+2}\right)-f_{2}^{\prime}\left(\rho_{n+1}\right)\right) \delta_{h} \rho_{n+1}+\int_{\Omega}\left(\mu_{n+1} g^{\prime}\left(\rho_{n+1}\right)-\mu_{n} g^{\prime}\left(\rho_{n}\right)\right) \delta_{h} \rho_{n+1} .
\end{aligned}
$$


The first term on the right-hand side of (4.13) is easily treated in the following way:

$$
-\int_{\Omega}\left(f_{2}^{\prime}\left(\rho_{n+2}\right)-f_{2}^{\prime}\left(\rho_{n+1}\right)\right) \delta_{h} \rho_{n+1} \leq c h \int_{\Omega}\left|\delta_{h} \rho_{n+1}\right|^{2}
$$

On the other hand, we have

$$
\begin{aligned}
& \int_{\Omega}\left(\mu_{n+1} g^{\prime}\left(\rho_{n+1}\right)-\mu_{n} g^{\prime}\left(\rho_{n}\right)\right) \delta_{h} \rho_{n+1} \\
& =\int_{\Omega} \mu_{n+1}\left(g^{\prime}\left(\rho_{n+1}\right)-g^{\prime}\left(\rho_{n}\right)\right) \delta_{h} \rho_{n+1}+\int_{\Omega}\left(\mu_{n+1}-\mu_{n}\right) g^{\prime}\left(\rho_{n}\right) \delta_{h} \rho_{n+1} \\
& \leq \operatorname{ch} \int_{\Omega} \mu_{n+1}\left|\delta_{h} \rho_{n}\right|\left|\delta_{h} \rho_{n+1}\right|+\int_{\Omega}\left(\mu_{n+1}-\mu_{n}\right) g^{\prime}\left(\rho_{n}\right) \delta_{h} \rho_{n+1} .
\end{aligned}
$$

Next, we deal with the last integral by using equation (2.27). Owing to our assumptions on $g$, we obtain

$$
\begin{aligned}
& \int_{\Omega}\left(\mu_{n+1}-\mu_{n}\right) g^{\prime}\left(\rho_{n}\right) \delta_{h} \rho_{n+1}=-h \int_{\Omega} \frac{g^{\prime}\left(\rho_{n}\right)}{1+2 \gamma_{n}}\left(\mu_{n+1} \delta_{h} \gamma_{n}-\Delta \mu_{n+1}\right) \delta_{h} \rho_{n+1} \\
& \leq \operatorname{ch} \int_{\Omega} \mu_{n+1}\left|\delta_{h} \rho_{n}\right|\left|\delta_{h} \rho_{n+1}\right|-h \int_{\Omega} \nabla \mu_{n+1} \cdot \nabla\left(\frac{g^{\prime}\left(\rho_{n}\right)}{1+2 \gamma_{n}} \delta_{h} \rho_{n+1}\right) \\
& \leq \operatorname{ch} \int_{\Omega} \mu_{n+1}\left|\delta_{h} \rho_{n}\right|\left|\delta_{h} \rho_{n+1}\right| \\
& \quad-h \int_{\Omega} \frac{g^{\prime}\left(\rho_{n}\right)}{1+2 \gamma_{n}} \nabla \mu_{n+1} \cdot \nabla \delta_{h} \rho_{n+1}-h \int_{\Omega} \delta_{h} \rho_{n+1} \nabla \mu_{n+1} \cdot \nabla \frac{g^{\prime}\left(\rho_{n}\right)}{1+2 \gamma_{n}} .
\end{aligned}
$$

We treat the last three terms separately. Thanks to the Hölder, Sobolev, and Young inequalities, and our assumption on $g$, we have for every $\sigma>0$

$$
\begin{aligned}
& h \int_{\Omega} \mu_{n+1}\left|\delta_{h} \rho_{n}\right|\left|\delta_{h} \rho_{n+1}\right| \leq c h\left\|\mu_{n+1}\right\|_{4}\left\|\delta_{h} \rho_{n}\right\|_{H}\left\|\delta_{h} \rho_{n+1}\right\|_{4} \\
& \leq \sigma h\left\|\delta_{h} \rho_{n+1}\right\|_{V}^{2}+\frac{c h}{\sigma}\left\|\mu_{n+1}\right\|_{V}^{2}\left\|\delta_{h} \rho_{n}\right\|_{H}^{2}, \\
& -h \int_{\Omega} \frac{g^{\prime}\left(\rho_{n}\right)}{1+2 \gamma_{n}} \nabla \mu_{n+1} \cdot \nabla \delta_{h} \rho_{n+1} \leq c h \int_{\Omega}\left|\nabla \mu_{n+1}\right|\left|\nabla \delta_{h} \rho_{n+1}\right| \\
& \leq \sigma h\left\|\nabla \delta_{h} \rho_{n+1}\right\|_{H}^{2}+\frac{c h}{\sigma}\left\|\nabla \mu_{n+1}\right\|_{H}^{2}, \\
& -h \int_{\Omega} \delta_{h} \rho_{n+1} \nabla \mu_{n+1} \cdot \nabla \frac{g^{\prime}\left(\rho_{n}\right)}{1+2 \gamma_{n}} \leq c h \int_{\Omega}\left|\delta_{h} \rho_{n+1}\right|\left|\nabla \mu_{n+1}\right|\left|\nabla \rho_{n}\right| \\
& \leq c h\left\|\delta_{h} \rho_{n+1}\right\|_{4}\left\|\nabla \mu_{n+1}\right\| H\left\|\nabla \rho_{n}\right\|_{4} \\
& \leq \sigma h\left\|\delta_{h} \rho_{n+1}\right\|_{V}^{2}+\frac{c h}{\sigma}\left\|\nabla \mu_{n+1}\right\|_{H}^{2}\left(\left\|\rho_{n}\right\|_{H}^{2}+\left\|\Delta \rho_{n}\right\|_{H}^{2}\right) .
\end{aligned}
$$

Now, we rewrite (4.8) as

$$
\left\|\Delta \rho_{n}\right\|_{H}^{2}+\left\|\xi_{n}\right\|_{H}^{2} \leq c\left(\left\|\delta_{h} \rho_{n-1}\right\|_{H}^{2}+\left\|\mu_{n-1}\right\|_{H}^{2}+1\right) \quad \text { for } n=1, \ldots, N,
$$

and note that we can allow the choice $n=0$ provided that we define

$$
\rho_{-1}:=\rho_{0} \text { and, e.g., } \mu_{-1}:=0 .
$$


Hence, we can improve (4.17). By using (4.3) and (4.7) as well, we have

$$
\begin{aligned}
& -h \int_{\Omega} \delta_{h} \rho_{n+1} \nabla \mu_{n+1} \cdot \nabla \frac{g^{\prime}\left(\rho_{n}\right)}{1+2 \gamma_{n}} \\
& \leq \sigma h\left\|\delta_{h} \rho_{n+1}\right\|_{V}^{2}+\frac{c h}{\sigma}\left\|\nabla \mu_{n+1}\right\|_{H}^{2}\left(\left\|\rho_{n}\right\|_{H}^{2}+\left\|\delta_{h} \rho_{n-1}\right\|_{H}^{2}+\left\|\mu_{n-1}\right\|_{H}^{2}+1\right) \\
& \leq \sigma h\left\|\delta_{h} \rho_{n+1}\right\|_{V}^{2}+\frac{c h}{\sigma}\left\|\nabla \mu_{n+1}\right\|_{H}^{2}\left(\left\|\delta_{h} \rho_{n-1}\right\|_{H}^{2}+1\right) .
\end{aligned}
$$

By recalling all these estimates, we see that (4.13) yields

$$
\begin{aligned}
& \frac{1}{2} \int_{\Omega}\left|\delta_{h} \rho_{n+1}\right|^{2}-\frac{1}{2} \int_{\Omega}\left|\delta_{h} \rho_{n}\right|^{2}+\frac{1}{2} \int_{\Omega}\left|\delta_{h} \rho_{n+1}-\delta_{h} \rho_{n}\right|^{2}+h \int_{\Omega}\left|\delta_{h} \nabla \rho_{n+1}\right|^{2} \\
& \leq c h \int_{\Omega}\left|\delta_{h} \rho_{n+1}\right|^{2}+3 \sigma h\left\|\delta_{h} \rho_{n+1}\right\|_{V}^{2}+\frac{c h}{\sigma}\left\|\mu_{n+1}\right\|_{V}^{2}\left\|\delta_{h} \rho_{n}\right\|_{H}^{2} \\
& \quad+\frac{c h}{\sigma}\left\|\nabla \mu_{n+1}\right\|_{H}^{2}\left(\left\|\delta_{h} \rho_{n-1}\right\|_{H}^{2}+1\right) .
\end{aligned}
$$

Now, just by changing the value of the constant $c$ in front of the first integral on the right-hand side, we can replace the last integral on the left-hand side by $\left\|\delta_{h} \rho_{n+1}\right\|_{V}^{2}$. Then, we choose $\sigma=1 / 4$ and rearrange. We obtain

$$
\begin{aligned}
& \frac{1}{2} \int_{\Omega}\left|\delta_{h} \rho_{n+1}\right|^{2}-\frac{1}{2} \int_{\Omega}\left|\delta_{h} \rho_{n}\right|^{2}+\frac{1}{2} \int_{\Omega}\left|\delta_{h} \rho_{n+1}-\delta_{h} \rho_{n}\right|^{2}+\frac{h}{4}\left\|\delta_{h} \rho_{n+1}\right\|_{V}^{2} \\
& \leq c h \int_{\Omega}\left|\delta_{h} \rho_{n+1}\right|^{2}+c h\left\|\mu_{n+1}\right\|_{V}^{2}\left\|\delta_{h} \rho_{n}\right\|_{H}^{2}+c h\left\|\nabla \mu_{n+1}\right\|_{H}^{2}\left(\left\|\delta_{h} \rho_{n-1}\right\|_{H}^{2}+1\right) .
\end{aligned}
$$

At this point, by assuming $m \leq N-1$, we sum over $n=0, \ldots, m-1$ and have

$$
\begin{aligned}
& \frac{1}{2} \int_{\Omega}\left|\delta_{h} \rho_{m}\right|^{2}+\frac{1}{2} \sum_{n=0}^{m-1} \int_{\Omega}\left|\delta_{h} \rho_{n+1}-\delta_{h} \rho_{n}\right|^{2}+\frac{h}{4} \sum_{n=0}^{m-1}\left\|\delta_{h} \rho_{n+1}\right\|_{V}^{2} \\
& \leq \frac{1}{2} \int_{\Omega}\left|\delta_{h} \rho_{0}\right|^{2}+c h \sum_{n=0}^{m-1} \int_{\Omega}\left|\delta_{h} \rho_{n+1}\right|^{2}+c h \sum_{n=0}^{m-1}\left\|\mu_{n+1}\right\|_{V}^{2}\left\|\delta_{h} \rho_{n}\right\|_{H}^{2} \\
& \quad+c h \sum_{n=0}^{m-1}\left\|\nabla \mu_{n+1}\right\|_{H}^{2}\left\|\delta_{h} \rho_{n-1}\right\|_{H}^{2}+c h \sum_{n=0}^{m-1}\left\|\nabla \mu_{n+1}\right\|_{H}^{2} .
\end{aligned}
$$

The second and the last terms on the right-hand side of (4.19) have been already estimated by (4.6) and (4.2), respectively. To treat the first term, we write (2.28) with $n=0$ and add $\Delta \rho_{0}$ to both sides. Then, we multiply the resulting equality by $\delta_{h} \rho_{0}$ and integrate over $\Omega$. After a rearrangement, owing to (2.4) and the assumptions on the initial data (see (2.7), in particular), we obtain:

$$
\begin{aligned}
& \int_{\Omega}\left|\delta_{h} \rho_{0}\right|^{2}+h \int_{\Omega}\left|\nabla \delta_{h} \rho_{0}\right|^{2}+\int_{\Omega}\left(f_{1}^{\prime}\left(\rho_{1}\right)-f_{1}^{\prime}\left(\rho_{0}\right)\right) \delta_{h} \rho_{0} \\
& =\int_{\Omega}\left(\Delta \rho_{0}+\mu_{0} g^{\prime}\left(\rho_{0}\right)-f_{1}^{\prime}\left(\rho_{0}\right)-f_{2}^{\prime}\left(\rho_{1}\right)\right) \delta_{h} \rho_{0} \leq c\left\|\delta_{h} \rho_{0}\right\|_{H} .
\end{aligned}
$$

As $\left(f_{1}^{\prime}\left(\rho_{1}\right)-f_{1}^{\prime}\left(\rho_{0}\right)\right) \delta_{h} \rho_{0} \geq 0$ due to the monotonicity of $f_{1}^{\prime}$, we immediately deduce that

$$
\left\|\delta_{h} \rho_{0}\right\|_{H}^{2}+h\left\|\nabla \delta_{h} \rho_{0}\right\|_{H}^{2} \leq c .
$$


In particular, the desired estimate for $\left\|\delta_{h} \rho_{0}\right\|_{H}$ is achieved. Therefore, on recalling that $\delta_{h} \rho_{-1}=0$ because $\rho_{-1}=\rho_{0}$, we see that (4.19) yields:

$$
\begin{aligned}
& \left\|\delta_{h} \rho_{m}\right\|_{H}^{2}+\sum_{n=0}^{m-1}\left\|\delta_{h} \rho_{n+1}-\delta_{h} \rho_{n}\right\|_{H}^{2}+h \sum_{n=0}^{m-1}\left\|\delta_{h} \rho_{n+1}\right\|_{V}^{2} \\
& \leq c+c h \sum_{n=0}^{m-1}\left\|\mu_{n+1}\right\|_{V}^{2}\left\|\delta_{h} \rho_{n}\right\|_{H}^{2}+c h \sum_{n=1}^{m-1}\left\|\nabla \mu_{n+1}\right\|_{H}^{2}\left\|\delta_{h} \rho_{n-1}\right\|_{H}^{2} \\
& \leq c+c h \sum_{n=0}^{m-1}\left\|\mu_{n+1}\right\|_{V}^{2}\left\|\delta_{h} \rho_{n}\right\|_{H}^{2}+c h \sum_{n=0}^{m-2}\left\|\nabla \mu_{n+2}\right\|_{H}^{2}\left\|\delta_{h} \rho_{n}\right\|_{H}^{2} \\
& \leq C_{1}+C_{2} h \sum_{n=0}^{m-1}\left(\left\|\mu_{n+1}\right\|_{V}^{2}+\left\|\mu_{n+2}\right\|_{V}^{2}\right)\left\|\delta_{h} \rho_{n}\right\|_{H}^{2}
\end{aligned}
$$

for $m=0, \ldots, N-1$. Hence, we can apply the discrete Gronwall lemma (see (2.38), where $N$ is to be replaced here by $N-1$ ) and deduce that

$$
\begin{aligned}
& \left\|\delta_{h} \rho_{m}\right\|_{H}^{2}+\sum_{n=0}^{m-1}\left\|\delta_{h} \rho_{n+1}-\delta_{h} \rho_{n}\right\|_{H}^{2}+h \sum_{n=0}^{m-1}\left\|\delta_{h} \rho_{n+1}\right\|_{V}^{2} \\
& \leq C_{1} \exp \left(C_{2} h \sum_{n=0}^{m-1}\left(\left\|\mu_{n+1}\right\|_{V}^{2}+\left\|\mu_{n+2}\right\|_{V}^{2}\right)\right)
\end{aligned}
$$

for $m=0, \ldots, N-1$. Owing to (4.2), we infer that

$$
\left\|\delta_{h} \rho_{m}\right\|_{H}^{2}+\sum_{n=0}^{m-1}\left\|\delta_{h} \rho_{n+1}-\delta_{h} \rho_{n}\right\|_{H}^{2}+h \sum_{n=0}^{m-1}\left\|\delta_{h} \rho_{n+1}\right\|_{V}^{2} \leq c \quad \text { for } m=0, \ldots, N-1 ;
$$

moreover, using the estimates of $\delta_{h} \rho_{0}$ and $\nabla \delta_{h} \rho_{0}$ given by (4.20), we conclude that

$$
\max _{m=0, \ldots, N-1}\left\|\delta_{h} \rho_{m}\right\|_{H}^{2}+\sum_{n=0}^{N-1}\left\|\delta_{h} \rho_{n}-\delta_{h} \rho_{n-1}\right\|_{H}^{2}+h \sum_{n=0}^{N-1}\left\|\delta_{h} \rho_{n}\right\|_{V}^{2} \leq c .
$$

In particular, (4.21) yields:

$$
\left\|\partial_{t} \widehat{\rho}_{h}\right\|_{L^{\infty}(0, T ; H) \cap L^{2}(0, T ; V)} \leq c .
$$

Fifth a priori estimate. We improve (4.10)-(4.11). Owing to (4.9), on using (4.21) in addition to previous estimates, we immediately obtain ( $c f$. also (2.7)) that

$$
\begin{aligned}
& \left\|\rho_{m}\right\|_{W}+\left\|\xi_{m}\right\|_{H} \leq c \quad \text { for } m=0, \ldots, N, \\
& \left\|\bar{\rho}_{h}\right\|_{L^{\infty}(0, T ; W)}^{2}+\left\|\underline{\rho}_{h}\right\|_{L^{\infty}(0, T ; W)}^{2}+\left\|\widehat{\rho}_{h}\right\|_{L^{\infty}(0, T ; W)}^{2} \leq c,
\end{aligned}
$$

as well as an estimate for, e.g., $\bar{\xi}_{h}$ in $L^{\infty}(0, T ; H)$.

Sixth a priori estimate. We rewrite (2.27) in the form

$$
\left(1+\gamma_{n}+\gamma_{n+1}\right) \delta_{h} \mu_{n}-\Delta \mu_{n+1}=-\mu_{n} \delta_{h} \gamma_{n}
$$


We test this equality by $\left(\mu_{n+1}-\mu_{n}\right)$, and integrate over $\Omega$. We obtain

$$
h \int_{\Omega}\left(1+\gamma_{n}+\gamma_{n+1}\right)\left|\delta_{h} \mu_{n}\right|^{2}+\int_{\Omega}\left(\nabla \mu_{n+1}-\nabla \mu_{n}\right) \cdot \nabla \mu_{n+1}=-h \int_{\Omega} \mu_{n} \delta_{h} \gamma_{n} \delta_{h} \mu_{n} .
$$

As $g$ is nonnegative and Lipschitz continuous, we infer that

$$
\begin{aligned}
& h \int_{\Omega}\left|\delta_{h} \mu_{n}\right|^{2}+\frac{1}{2} \int_{\Omega}\left|\nabla \mu_{n+1}\right|^{2}-\frac{1}{2} \int_{\Omega}\left|\nabla \mu_{n}\right|^{2}+\frac{1}{2} \int_{\Omega}\left|\nabla\left(\mu_{n+1}-\mu_{n}\right)\right|^{2} \\
& \leq c h \int_{\Omega} \mu_{n}\left|\delta_{h} \rho_{n}\right|\left|\delta_{h} \mu_{n}\right| \leq c h\left\|\mu_{n}\right\|_{4}\left\|\delta_{h} \rho_{n}\right\|_{4}\left\|\delta_{h} \mu_{n}\right\|_{2} \\
& \leq \frac{h}{2}\left\|\delta_{h} \mu_{n}\right\|_{H}^{2}+c h\left\|\delta_{h} \rho_{n}\right\|_{V}^{2}\left(\left\|\nabla \mu_{n}\right\|_{H}^{2}+\left\|\mu_{n}\right\|_{H}^{2}\right) \\
& \leq \frac{h}{2}\left\|\delta_{h} \mu_{n}\right\|_{H}^{2}+c h\left\|\delta_{h} \rho_{n}\right\|_{V}^{2}\left\|\nabla \mu_{n}\right\|_{H}^{2}+c h\left\|\delta_{h} \rho_{n}\right\|_{V}^{2},
\end{aligned}
$$

the last inequality being due to (4.2). By rearranging and summing over $n=0, \ldots, m-1$ with $1 \leq m \leq N$, we get:

$$
\begin{aligned}
& \frac{h}{2} \sum_{n=0}^{m-1}\left\|\delta_{h} \mu_{n}\right\|_{H}^{2}+\frac{1}{2}\left\|\nabla \mu_{m}\right\|_{H}^{2}+\frac{h^{2}}{2} \sum_{n=0}^{m-1}\left\|\nabla \delta_{h} \mu_{n}\right\|_{H}^{2} \\
& \leq \frac{1}{2}\left\|\nabla \mu_{0}\right\|_{H}^{2}+c h \sum_{n=0}^{m-1}\left\|\delta_{h} \rho_{n}\right\|_{V}^{2}\left\|\nabla \mu_{n}\right\|_{H}^{2}+c h \sum_{n=0}^{m-1}\left\|\delta_{h} \rho_{n}\right\|_{V}^{2} \\
& \leq c+c h \sum_{n=0}^{m-1}\left\|\delta_{h} \rho_{n}\right\|_{V}^{2}\left\|\nabla \mu_{n}\right\|_{H}^{2}
\end{aligned}
$$

where we have used (4.21). Now, we first apply the discrete Gronwall lemma (2.38) and then account for (4.21) once more. We obtain, for $m=1, \ldots, N$,

$$
h \sum_{n=0}^{m-1}\left\|\delta_{h} \mu_{n}\right\|_{H}^{2}+\left\|\nabla \mu_{m}\right\|_{H}^{2}+h^{2} \sum_{n=0}^{m-1}\left\|\nabla \delta_{h} \mu_{n}\right\|_{H}^{2} \leq c .
$$

Next, by (2.27), the Hölder and Sobolev inequalities and the Lipschitz continuity of $g$, we infer that

$$
\begin{aligned}
& \left\|\Delta \mu_{n+1}\right\|_{H} \leq c\left(\left\|\delta_{h} \mu_{n}\right\|_{H}+\left\|\mu_{n+1} \delta_{h} \gamma_{n}\right\|_{H}\right) \leq c\left(\left\|\delta_{h} \mu_{n}\right\|_{H}+\left\|\mu_{n+1}\right\|_{4}\left\|\delta_{h} \gamma_{n}\right\|_{4}\right) \\
& \leq c\left(\left\|\delta_{h} \mu_{n}\right\|_{H}+\left\|\mu_{n+1}\right\|_{4}\left\|\delta_{h} \rho_{n}\right\|_{4}\right) \leq c\left(\left\|\delta_{h} \mu_{n}\right\|_{H}+\left\|\mu_{n+1}\right\|_{V}\left\|\delta_{h} \rho_{n}\right\|_{V}\right) ;
\end{aligned}
$$

note that in the last product we can ignore the factor $\left\|\mu_{n+1}\right\|_{V}$, due to (4.3) and (4.25), provided we update the last value of $c$. By squaring, summing up, and multiplying by $h$, we thus obtain for $m=1, \ldots, N$ the estimate

$$
h \sum_{n=0}^{m-1}\left\|\Delta \mu_{n+1}\right\|_{H}^{2} \leq c h \sum_{n=0}^{m-1}\left\|\delta_{h} \mu_{n}\right\|_{H}^{2}+c h \sum_{n=0}^{m-1}\left\|\delta_{h} \rho_{n}\right\|_{V}^{2},
$$

and we can replace the $H$-norm of $\Delta \mu_{n+1}$ by the $W$-norm of $\mu_{n+1}$ thanks to (4.2). We collect this and (4.25) and account for (4.21) and $\mu_{0} \in V$. We have:

$$
h \sum_{n=0}^{N-1}\left\|\delta_{h} \mu_{n}\right\|_{H}^{2}+\max _{n=0, \ldots, N}\left\|\nabla \mu_{n}\right\|_{H}^{2}+h \sum_{n=0}^{N-1}\left\|\mu_{n+1}\right\|_{W}^{2} \leq c,
$$


so that

$$
\left\|\partial_{t} \widehat{\mu}_{h}\right\|_{L^{2}(0, T ; H)}+\left\|\bar{\mu}_{h}\right\|_{L^{\infty}(0, T ; V) \cap L^{2}(0, T ; W)}+\left\|\underline{\mu}_{h}\right\|_{L^{\infty}(0, T ; V)}+\left\|\widehat{\mu}_{h}\right\|_{L^{\infty}(0, T ; V)} \leq c .
$$

We note that (4.25) also gives the non-sharp estimate

$$
h\left\|\nabla \partial_{t} \widehat{\mu}_{h}\right\|_{L^{2}(0, T ; H)}^{2} \leq c .
$$

Limit and conclusion. By standard weak compactness results, we find some convergent subsequence for the interpolants. Therefore, in principle, it is understood that the convergence that we refer to holds for a subsequence. However, once we prove that the limit we find is the solution $(\mu, \rho)$ to problem $(2.12)-(2.14)$, then the whole family of interpolants is convergent, due to uniqueness. For the reader's convenience, we select some estimates among those we have proved in the previous steps. These are:

$$
\begin{aligned}
& \left\|\bar{\mu}_{h}\right\|_{L^{\infty}(0, T ; V) \cap L^{2}(0, T ; W)}+\left\|\underline{\mu}_{h}\right\|_{L^{\infty}(0, T ; V)}+\left\|\widehat{\mu}_{h}\right\|_{L^{\infty}(0, T ; V)} \leq c \\
& \left\|\bar{\rho}_{h}\right\|_{L^{\infty}(0, T ; W)}+\left\|\underline{\rho}_{h}\right\|_{L^{\infty}(0, T ; W)}+\left\|\widehat{\rho}_{h}\right\|_{L^{\infty}(0, T ; W)} \leq c \\
& \left\|\partial_{t} \widehat{\mu}_{h}\right\|_{L^{2}(0, T ; H)}+\left\|\partial_{t} \widehat{\rho}_{h}\right\|_{L^{\infty}(0, T ; H) \cap L^{2}(0, T ; V)} \leq c .
\end{aligned}
$$

Now, we observe that (4.31) and (2.25) imply that

$$
\begin{aligned}
& \left\|\bar{\mu}_{h}-\widehat{\mu}_{h}\right\|_{L^{2}(0, T ; H)}+\left\|\underline{\mu}_{h}-\widehat{\mu}_{h}\right\|_{L^{2}(0, T ; H)} \leq c h \\
& \left\|\bar{\rho}_{h}-\widehat{\rho}_{h}\right\|_{L^{2}(0, T ; V)}+\left\|\underline{\rho}_{h}-\widehat{\rho}_{h}\right\|_{L^{2}(0, T ; V)} \leq c h .
\end{aligned}
$$

This yields, in particular, that the weak limits we find for $\bar{\mu}_{h}, \underline{\mu}_{h}$, and $\widehat{\mu}_{h}$, by using (4.29) and weak compactness results coincide and that the same happens for $\bar{\rho}_{h}, \underline{\rho}_{h}$, and $\widehat{\rho}_{h}$. Therefore, we can conclude that some functions $\mu$ and $\rho$ exist such that

$$
\begin{array}{cl}
\bar{\mu}_{h}, \underline{\mu}_{h}, \widehat{\mu}_{h} \rightarrow \mu & \text { weakly star in } L^{\infty}(0, T ; V), \\
\bar{\mu}_{h} \rightarrow \mu & \text { weakly in } L^{2}(0, T ; W), \\
\bar{\rho}_{h}, \underline{\rho}_{h}, \widehat{\rho}_{h} \rightarrow \rho & \text { weakly star in } L^{\infty}(0, T ; W), \\
\partial_{t} \widehat{\mu}_{h} \rightarrow \partial_{t} \mu & \text { weakly in } L^{2}(0, T ; H), \\
\partial_{t} \widehat{\rho}_{h} \rightarrow \partial_{t} \rho & \text { weakly star in } L^{\infty}(0, T ; H) \cap L^{2}(0, T ; V) .
\end{array}
$$

Now we prove that $(\mu, \rho)$ satisfies (2.8)-(2.11) and solves problem (2.12)-(2.14).

We remark that the topology alluded to in the statement of Theorem 2.4 is precisely the topology associated with the convergences specified in (4.34)-(4.38). Clearly, (2.8)-(2.10) are fulfilled. Moreover, the Cauchy conditions (2.14) are satisfied, because $\left(\widehat{\mu}_{h}, \widehat{\rho}_{h}\right)$ converges to $(\mu, \rho)$ at least weakly in $C^{0}([0, T] ; H)$. Therefore, it remains to check that (2.11) holds and that equations (2.12)-(2.13) are satisfied. To do that, we read the discrete problem (2.27)-(2.28) in terms of the interpolants. We have:

$$
\begin{aligned}
& \left(1+2 \underline{\gamma}_{h}\right) \partial_{t} \widehat{\mu}_{h}+\bar{\mu}_{h} \partial_{t} \widehat{\gamma}_{h}-\Delta \bar{\mu}_{h}=0, \\
& \partial_{t} \widehat{\rho}_{h}-\Delta \bar{\rho}_{h}+f^{\prime}\left(\bar{\rho}_{h}\right)=\underline{\mu}_{h} g^{\prime}\left(\underline{\rho}_{h}\right) .
\end{aligned}
$$

Hence, the main problem consists in identifying correctly the limits of the nonlinear terms and those of the products. To this end, we recover some strong convergence (without looking for sharpness, since it is not necessary). We first recall that the embeddings $V \subset H$ and $W \subset C^{0}(\bar{\Omega})$ are compact, so that we can apply [32] (Sect. 8, Cor. 4) and deduce that

$$
\begin{array}{ll}
\widehat{\mu}_{h} \rightarrow \mu & \text { strongly in } C^{0}([0, T] ; H) \text { and a.e. in } Q, \\
\widehat{\rho}_{h} \rightarrow \rho & \text { strongly in } C^{0}\left([0, T] ; C^{0}(\bar{\Omega})\right)=C^{0}(\bar{Q}) .
\end{array}
$$


By combining this with (4.32) and (4.33), we infer that

$$
\bar{\mu}_{h}, \underline{\mu}_{h} \rightarrow \mu \quad \text { and } \quad \bar{\rho}_{h}, \underline{\rho}_{h} \rightarrow \rho \text { strongly in } L^{2}(0, T ; H) \text { and a.e. in } Q \text {. }
$$

We point out that a.e.-convergence actually holds for a subsequence. As $f_{2}^{\prime}, g$, and $g^{\prime}$ are Lipschitz continuous on $[0,1]$, we deduce that

$$
\phi\left(\bar{\rho}_{h}\right), \phi\left(\underline{\rho}_{h}\right) \rightarrow \phi(\rho) \quad \text { strongly in } L^{2}(0, T ; H) \quad \text { for } \phi=f_{2}^{\prime}, g, g^{\prime}
$$

On the other hand, by comparison in $(4.40)$, we see that $f_{1}^{\prime}\left(\bar{\rho}_{h}\right)$ remains bounded in $L^{\infty}(0, T ; H)$, so that $f_{1}^{\prime}\left(\bar{\rho}_{h}\right)$ converges (for a subsequence) to some $\xi$ in the weak star topology of such a space. As $f_{1}^{\prime}$ induces a maximal monotone operator on $L^{2}(Q) \times L^{2}(Q)\left(c f ., e . g .,[10]\right.$, Ex. 2.3.3, p. 25), $f_{1}^{\prime}\left(\bar{\rho}_{h}\right) \rightarrow \xi$ and $\bar{\rho}_{h} \rightarrow \rho$ weakly in $L^{2}(Q)$, and

$$
\limsup _{h \searrow 0} \int_{Q} f_{1}^{\prime}\left(\bar{\rho}_{h}\right) \bar{\rho}_{h} \leq \int_{Q} \xi \rho,
$$

owing to standard results in the theory of maximal monotone operators (one may see [10], Prop. 2.5, p. 27), we deduce that $0<\rho<1$ and $\xi=f_{1}^{\prime}(\rho)$ a.e. in $Q$. In particular, (2.11) holds. Furthermore, we also have

$$
\left|\partial_{t} \widehat{\gamma}_{h}\right|=\left|\delta_{h} \gamma_{n}\right|=\left|\delta_{h} g\left(\rho_{n}\right)\right| \leq c\left|\delta_{h} \rho_{n}\right|=c\left|\partial_{t} \widehat{\rho}_{h}\right| \quad \text { a.e. in } I_{n+1}, \text { for } n=0, \ldots, N-1,
$$

so that (4.31) yields an estimate of $\partial_{t} \widehat{\gamma}_{h}$ in $L^{\infty}(0, T ; H)$. Hence, thanks to $(2.24)$, we have

$$
\left\|\widehat{\gamma}_{h}-\bar{\gamma}_{h}\right\|_{L^{\infty}(0, T ; H)} \leq c h\left\|\partial_{t} \widehat{\gamma}_{h}\right\|_{L^{\infty}(0, T ; H)} \leq c h,
$$

whence even $\widehat{\gamma}_{h}$ converges to $g(\rho)$, e.g., strongly in $L^{2}(Q)$. Then, we deduce that

$$
\partial_{t} \widehat{\gamma}_{h} \rightarrow \partial_{t} g(\rho) \quad \text { weakly star in } L^{\infty}(0, T ; H) .
$$

Finally, as to the limits of the products in (4.39)-(4.40), we can infer that

$$
\underline{\gamma}_{h} \partial_{t} \widehat{\mu}_{h} \rightarrow g(\rho) \partial_{t} \mu, \quad \bar{\mu}_{h} \partial_{t} \widehat{\gamma}_{h} \rightarrow \mu \partial_{t} g(\rho), \quad \bar{\mu}_{h} g^{\prime}\left(\underline{\rho}_{h}\right) \rightarrow \mu g^{\prime}(\rho), \quad \text { weakly in } L^{1}(Q) .
$$

Therefore, (2.12)-(2.13) follow from (4.39)-(4.40), and the proof is complete. In particular, let us stress that the so found pair $(\mu, \rho)$ solves $(2.8)-(2.14)$ and then it must coincide with the unique solution $(\mu, \rho)$ of the continuous problem given by Theorem 2.1 .

As a by-product of the above proof, it turns out that

$$
\rho_{\bullet} \leq \widehat{\rho}_{h}, \bar{\rho}_{h}, \underline{\rho}_{h} \leq \rho^{\bullet} \text { in } Q, \text { for some } \rho_{\bullet}, \rho^{\bullet} \in(0,1),
$$

provided that $h$ is small enough. Indeed, take $\rho_{\bullet} \in\left(0, \rho_{*}\right)$ and $\rho^{\bullet} \in\left(\rho^{*}, 1\right)$, with $\rho_{*}, \rho^{*} \in(0,1)$ given by Theorem 2.1. That (4.46) holds for $\widehat{\rho}_{h}$ follows from the uniform convergence given by (4.42). This means that the same bounds hold for $\rho_{n}, n=0, \ldots, N$ (where $\left(\rho_{n}\right)_{n=0}^{N}$ is the vector associated with $\widehat{\rho}_{h}$ ), and hence also for $\bar{\rho}_{h}$ and $\underline{\rho}_{h}$.

\section{Proof of Theorem 2.5}

In this section, we prove Theorem 2.5. It is understood that $h$ is as small as needed; oftentimes, we do not pause and quantify such smallness precisely. First of all, we remind the reader that the interpolants $\widehat{\rho}_{h}, \underline{\rho}_{h}$, and $\bar{\rho}_{h}$ are uniformly far for 0 and 1 (see (4.46) and the subsequent lines). Therefore, without loss of generality, we can assume that the derivative function $f^{\prime}$ is Lipschitz continuous. We need additional a priori estimates. 
Auxiliary a priori estimates. We prepare an estimate for $\left\|\nabla \delta_{h} \rho_{0}\right\|_{H}$. To this end, we notice that (2.28) with $n=0$ can be written as

$$
\delta_{h} \rho_{0}-h \Delta \delta_{h} \rho_{0}=f^{\prime}\left(\rho_{0}\right)-f^{\prime}\left(\rho_{1}\right)-\psi_{0},
$$

where $\psi_{0}:=-\Delta \rho_{0}+f^{\prime}\left(\rho_{0}\right)-\mu_{0} g^{\prime}\left(\rho_{0}\right)$. As $\psi_{0} \in V$ by (2.33), we can test (5.1) by $-\Delta \delta_{h} \rho_{0}$ and integrate by parts. In view of the Lipschitz continuity of $f^{\prime}$, we find out that

$$
\begin{aligned}
& \left\|\nabla \delta_{h} \rho_{0}\right\|_{H}^{2}+h\left\|\Delta \delta_{h} \rho_{0}\right\|_{H}^{2} \leq c h\left\|\delta_{h} \rho_{0}\right\|_{H}\left\|\Delta \delta_{h} \rho_{0}\right\|_{H}+\left\|\nabla \psi_{0}\right\|_{H}\left\|\nabla \delta_{h} \rho_{0}\right\|_{H} \\
& \leq \frac{h}{2}\left\|\Delta \delta_{h} \rho_{0}\right\|_{H}^{2}+c h\left\|\delta_{h} \rho_{0}\right\|_{H}^{2}+\frac{1}{2}\left\|\nabla \delta_{h} \rho_{0}\right\|_{H}^{2}+c .
\end{aligned}
$$

By accounting for (4.20), we obtain the desired estimate

$$
\left\|\nabla \delta_{h} \rho_{0}\right\|_{H} \leq c .
$$

Let us come now to the basic estimate we need. We write (2.28) with $(n+1)$ in place of $n$, and take the difference between the so-obtained equality and (2.28) itself. Then, we multiply this difference by $-\Delta \delta_{h} \rho_{n+1}$ and integrate over $\Omega$. We easily have, for $n=0, \ldots, N-2$, that

$$
\begin{aligned}
& \int_{\Omega}\left(\nabla \delta_{h} \rho_{n+1}-\nabla \delta_{h} \rho_{n}\right) \cdot \nabla \delta_{h} \rho_{n+1}+\int_{\Omega}\left(\Delta \rho_{n+2}-\Delta \rho_{n+1}\right) \Delta \delta_{h} \rho_{n+1} \\
& =-\int_{\Omega}\left(f^{\prime}\left(\rho_{n+2}\right)-f^{\prime}\left(\rho_{n+1}\right)\right)\left(-\Delta \delta_{h} \rho_{n+1}\right) \\
& \quad+\int_{\Omega}\left(\mu_{n+1} g^{\prime}\left(\rho_{n+1}\right)-\mu_{n} g^{\prime}\left(\rho_{n}\right)\right)\left(-\Delta \delta_{h} \rho_{n+1}\right) .
\end{aligned}
$$

By the elementary identity (2.36), the first integral is equal to

$$
\frac{1}{2} \int_{\Omega}\left|\nabla \delta_{h} \rho_{n+1}\right|^{2}-\frac{1}{2} \int_{\Omega}\left|\nabla \delta_{h} \rho_{n}\right|^{2}+\frac{1}{2} \int_{\Omega}\left|\nabla \delta_{h} \rho_{n+1}-\nabla \delta_{h} \rho_{n}\right|^{2} .
$$

On the other hand, we obviously have that

$$
\int_{\Omega}\left(\Delta \rho_{n+2}-\Delta \rho_{n+1}\right) \Delta \delta_{h} \rho_{n+1}=h \int_{\Omega}\left|\Delta \delta_{h} \rho_{n+1}\right|^{2} .
$$

Now, we deal with the right-hand side of (5.3). By Lipschitz continuity, we deduce that

$$
\begin{aligned}
& -\int_{\Omega}\left(f^{\prime}\left(\rho_{n+2}\right)-f^{\prime}\left(\rho_{n+1}\right)\right)\left(-\Delta \delta_{h} \rho_{n+1}\right) \leq c \int_{\Omega}\left|\rho_{n+2}-\rho_{n+1}\right|\left|\Delta \delta_{h} \rho_{n+1}\right| \\
& \leq c h \int_{\Omega}\left|\delta_{h} \rho_{n+1}\right|\left|\Delta \delta_{h} \rho_{n+1}\right| \leq \frac{h}{4} \int_{\Omega}\left|\Delta \delta_{h} \rho_{n+1}\right|^{2}+c h \int_{\Omega}\left|\delta_{h} \rho_{n+1}\right|^{2} .
\end{aligned}
$$

As far as the last term of (5.3) is concerned, we combine the above elementary argument with the Hölder and Young inequalities and the Sobolev embedding $V \subset L^{4}(\Omega)$. We find:

$$
\begin{aligned}
& \int_{\Omega}\left(\mu_{n+1} g^{\prime}\left(\rho_{n+1}\right)-\mu_{n} g^{\prime}\left(\rho_{n}\right)\right)\left(-\Delta \delta_{h} \rho_{n+1}\right) \\
& \leq c \int_{\Omega}\left(\mu_{n+1}\left|\rho_{n+1}-\rho_{n}\right|+\left|\mu_{n+1}-\mu_{n}\right|\right)\left|\Delta \delta_{h} \rho_{n+1}\right| \\
& \leq c h\left(\left\|\mu_{n+1}\right\|_{4}\left\|\delta_{h} \rho_{n}\right\|_{4}+\left\|\delta_{h} \mu_{n}\right\|_{H}\right)\left\|\Delta \delta_{h} \rho_{n+1}\right\|_{H} \\
& \leq \frac{h}{4} \int_{\Omega}\left|\Delta \delta_{h} \rho_{n+1}\right|^{2}+c h\left\|\mu_{n+1}\right\|_{V}^{2}\left\|\delta_{h} \rho_{n}\right\|_{V}^{2}+\operatorname{ch}\left\|\delta_{h} \mu_{n}\right\|_{H}^{2} .
\end{aligned}
$$


By collecting the inequalities we have obtained, we see that (5.3) yields:

$$
\begin{aligned}
& \frac{1}{2} \int_{\Omega}\left|\nabla \delta_{h} \rho_{n+1}\right|^{2}-\frac{1}{2} \int_{\Omega}\left|\nabla \delta_{h} \rho_{n}\right|^{2}+\frac{1}{2} \int_{\Omega}\left|\nabla \delta_{h} \rho_{n+1}-\nabla \delta_{h} \rho_{n}\right|^{2}+\frac{h}{2} \int_{\Omega}\left|\Delta \delta_{h} \rho_{n+1}\right|^{2} \\
& \leq c h \int_{\Omega}\left|\delta_{h} \rho_{n+1}\right|^{2}+c h\left\|\mu_{n+1}\right\|_{V}^{2}\left\|\delta_{h} \rho_{n}\right\|_{V}^{2}+\operatorname{ch}\left\|\delta_{h} \mu_{n}\right\|_{H}^{2} .
\end{aligned}
$$

At this point, we sum over $n=0, \ldots, m-1$, with $1 \leq m \leq N-1$, and deduce that

$$
\begin{aligned}
& \frac{1}{2} \int_{\Omega}\left|\nabla \delta_{h} \rho_{m}\right|^{2}+\frac{1}{2} \sum_{n=0}^{m-1} \int_{\Omega}\left|\nabla \delta_{h} \rho_{n+1}-\nabla \delta_{h} \rho_{n}\right|^{2}+\frac{h}{2} \sum_{n=0}^{m-1} \int_{\Omega}\left|\Delta \delta_{h} \rho_{n+1}\right|^{2} \\
& \leq \frac{1}{2} \int_{\Omega}\left|\nabla \delta_{h} \rho_{0}\right|^{2}+c h \sum_{n=0}^{N-2}\left\|\delta_{h} \rho_{n+1}\right\|_{H}^{2} \\
& \quad+c \max _{n=0, \ldots, N-1}\left\|\mu_{n+1}\right\|_{V}^{2} h \sum_{n=0}^{N-1}\left\|\delta_{h} \rho_{n}\right\|_{V}^{2}+c h \sum_{n=0}^{N-1}\left\|\delta_{h} \mu_{n}\right\|_{H}^{2} .
\end{aligned}
$$

The first term on the right-hand side of (5.4) is estimated by (5.2); all other terms on the right-hand side have been estimated already ( $c f .(4.22)$ and (4.27)). Therefore, by recalling also (4.21), we conclude that

$$
\begin{aligned}
& \max _{m=0, \ldots, N-1}\left\|\delta_{h} \rho_{m}\right\|_{V}^{2}+h \sum_{n=0}^{N-2}\left\|\Delta \delta_{h} \rho_{n+1}\right\|_{H}^{2} \leq c, \\
& \left\|\partial_{t} \widehat{\rho}_{h}\right\|_{L^{\infty}(0, T ; V)}+\left\|\Delta \partial_{t} \widehat{\rho}_{h}\right\|_{L^{2}(0, T ; H)} \leq c .
\end{aligned}
$$

Consequence. In view of the regularity theory for elliptic equations and the continuous embedding $W \subset$ $L^{\infty}(\Omega)$, we derive from (5.6) that

$$
\left\|\partial_{t} \widehat{\rho}_{h}\right\|_{L^{2}(0, T ; W)} \leq c \quad \text { and } \quad\left\|\partial_{t} \widehat{\rho}_{h}\right\|_{L^{2}\left(0, T ; L^{\infty}(\Omega)\right)} \leq c .
$$

Moreover, as the second (5.7) means an estimate of the difference quotients associated to the vector $\left(\rho_{n}\right)_{n=0}^{N}$, and as $g$ is Lipschitz continuous, a similar estimate holds for the vector $\left(g\left(\rho_{n}\right)\right)_{n=0}^{N}$ (see (4.1)), and we infer that

$$
\left\|\partial_{t} \widehat{\gamma}_{h}\right\|_{L^{2}\left(0, T ; L^{\infty}(\Omega)\right)} \leq c .
$$

Furthermore, by applying (2.25), we see that (5.6) also implies that

$$
\left\|\Delta\left(\bar{\rho}_{h}-\widehat{\rho}_{h}\right)\right\|_{L^{2}(0, T ; H)} \leq c h .
$$

Proof of Theorem 2.5. A possible strategy could be the following: to multiply the difference between (4.39) and $(2.12)$ by $\left(\widehat{\mu}_{h}-\mu\right)$, and the difference between (4.40) and (2.13) by $\partial_{t}\left(\widehat{\rho}_{h}-\rho\right)$; then, to sum up and start estimating. However, in order to split calculations and give more transparence to the proof, we prefer to proceed with those pairs of equation separately, and collect the inequalities we obtain later on. So, we first consider just one couple, for instance, (4.40) and (2.13). We multiply their difference by $\partial_{t}\left(\widehat{\rho}_{h}-\rho\right)$, integrate over $Q_{t}$, where 
$t \in(0, T)$ is arbitrary, and add the same integral to both sides for convenience. We obtain:

$$
\begin{aligned}
\int_{0}^{t} \int_{\Omega}\left|\partial_{t}\left(\widehat{\rho}_{h}-\rho\right)\right|^{2}+ & \frac{1}{2}\left\|\left(\widehat{\rho}_{h}-\rho\right)(t)\right\|_{V}^{2} \\
= & \int_{0}^{t} \int_{\Omega}\left\{-\Delta\left(\widehat{\rho}_{h}-\bar{\rho}_{h}\right)-\left(f^{\prime}\left(\bar{\rho}_{h}\right)-f^{\prime}(\rho)\right)\right. \\
& \left.+g^{\prime}\left(\underline{\rho}_{h}\right)\left(\underline{\mu}_{h}-\mu\right)+\mu\left(g^{\prime}\left(\underline{\rho}_{h}\right)-g^{\prime}(\rho)\right)+\left(\widehat{\rho}_{h}-\rho\right)\right\} \partial_{t}\left(\widehat{\rho}_{h}-\rho\right) \\
\leq & \frac{1}{2} \int_{0}^{t} \int_{\Omega}\left|\partial_{t}\left(\widehat{\rho}_{h}-\rho\right)\right|^{2} \\
& +c \int_{0}^{t} \int_{\Omega}\left\{\left|\Delta\left(\widehat{\rho}_{h}-\bar{\rho}_{h}\right)\right|^{2}+\left|\bar{\rho}_{h}-\rho\right|^{2}+\left|\underline{\mu}_{h}-\mu\right|^{2}+\left|\underline{\rho}_{h}-\rho\right|^{2}+\left|\widehat{\rho}_{h}-\rho\right|^{2}\right\} .
\end{aligned}
$$

In the above inequality, we have used the Lipschitz continuity of $f^{\prime}$ and $g^{\prime}$, and the boundedness of $\mu$. Now, we estimate the last integral of (5.10). Thanks to (5.9), we have that

$$
\int_{0}^{t} \int_{\Omega}\left|\Delta\left(\widehat{\rho}_{h}-\bar{\rho}_{h}\right)\right|^{2} \leq c h^{2} .
$$

On the other hand, owing to (4.33), we obtain:

$$
\begin{aligned}
\int_{0}^{t} \int_{\Omega}\left(\left|\bar{\rho}_{h}-\rho\right|^{2}+\left|\underline{\rho}_{h}-\rho\right|^{2}+\left|\widehat{\rho}_{h}-\rho\right|^{2}\right) & \leq c \int_{0}^{t} \int_{\Omega}\left(\left|\bar{\rho}_{h}-\widehat{\rho}_{h}\right|^{2}+\left|\underline{\rho}_{h}-\widehat{\rho}_{h}\right|^{2}+\left|\widehat{\rho}_{h}-\rho\right|^{2}\right) \\
& \leq c h^{2}+c \int_{0}^{t} \int_{\Omega}\left|\widehat{\rho}_{h}-\rho\right|^{2} .
\end{aligned}
$$

Similarly, we have, by (4.32), that

$$
\int_{0}^{t} \int_{\Omega}\left|\underline{\mu}_{h}-\mu\right|^{2} \leq c \int_{0}^{t} \int_{\Omega}\left(\left|\underline{\mu}_{h}-\widehat{\mu}_{h}\right|^{2}+\left|\widehat{\mu}_{h}-\mu\right|^{2}\right) \leq c h^{2}+c \int_{0}^{t} \int_{\Omega}\left|\widehat{\mu}_{h}-\mu\right|^{2} .
$$

By collecting the above inequalities, we see that (5.10) and the Gronwall lemma yield:

$$
\int_{0}^{t} \int_{\Omega}\left|\partial_{t}\left(\widehat{\rho}_{h}-\rho\right)\right|^{2}+\left\|\left(\widehat{\rho}_{h}-\rho\right)(t)\right\|_{V}^{2} \leq c\left\{h^{2}+\int_{0}^{t} \int_{\Omega}\left|\widehat{\rho}_{h}-\rho\right|^{2}+\int_{0}^{t} \int_{\Omega}\left|\widehat{\mu}_{h}-\mu\right|^{2}\right\}
$$

for every $t \in[0, T]$. Now, we deal with equations (4.39) and (2.12). For the reader's convenience, by recalling that $\bar{\gamma}_{h}=g\left(\bar{\rho}_{h}\right)$ and $\underline{\gamma}_{h}=g\left(\underline{\rho}_{h}\right)$ (see $(4.1)$ ), we rewrite the former in a different way, namely,

$$
\left(1+2 g\left(\underline{\rho}_{h}\right)\right) \partial_{t} \widehat{\mu}_{h}+\bar{\mu}_{h} \partial_{t} \widehat{\gamma}_{h}-\Delta \bar{\mu}_{h}=0 .
$$

Next, we take the difference between (5.12) and (2.12) and write it as

$$
\begin{aligned}
\left(1+2 g\left(\widehat{\rho}_{h}\right)\right) \partial_{t}\left(\widehat{\mu}_{h}-\mu\right)-\Delta\left(\widehat{\mu}_{h}-\mu\right)+\left(\widehat{\mu}_{h}-\mu\right)= & -2 \partial_{t} \mu\left(g\left(\widehat{\rho}_{h}\right)-g(\rho)\right)-\partial_{t} \widehat{\gamma}_{h}\left(\bar{\mu}_{h}-\mu\right)-\mu \partial_{t}\left(\widehat{\gamma}_{h}-g(\rho)\right) \\
& +2\left(g\left(\widehat{\rho}_{h}\right)-g\left(\underline{\rho}_{h}\right)\right) \partial_{t} \widehat{\mu}_{h}-\Delta\left(\widehat{\mu}_{h}-\bar{\mu}_{h}\right)+\left(\widehat{\mu}_{h}-\mu\right) .
\end{aligned}
$$

Finally, we multiply this equality by $\left(\widehat{\mu}_{h}-\mu\right)$ and obtain the following identity:

$$
\begin{aligned}
\partial_{t} & \left\{\left(\frac{1}{2}+g\left(\widehat{\rho}_{h}\right)\right)\left(\widehat{\mu}_{h}-\mu\right)^{2}\right\}-\Delta\left(\widehat{\mu}_{h}-\mu\right)\left(\widehat{\mu}_{h}-\mu\right)+\left(\widehat{\mu}_{h}-\mu\right)^{2} \\
=\partial_{t} g & \left(\widehat{\rho}_{h}\right)\left(\widehat{\mu}_{h}-\mu\right)^{2}-2 \partial_{t} \mu\left(g\left(\widehat{\rho}_{h}\right)-g(\rho)\right)\left(\widehat{\mu}_{h}-\mu\right) \\
& -\partial_{t} \widehat{\gamma}_{h}\left(\bar{\mu}_{h}-\mu\right)\left(\widehat{\mu}_{h}-\mu\right)-\mu \partial_{t}\left(\widehat{\gamma}_{h}-g(\rho)\right)\left(\widehat{\mu}_{h}-\mu\right) \\
& +2\left(g\left(\widehat{\rho}_{h}\right)-g\left(\underline{\rho}_{h}\right)\right) \partial_{t} \widehat{\mu}_{h}\left(\widehat{\mu}_{h}-\mu\right)-\Delta\left(\widehat{\mu}_{h}-\bar{\mu}_{h}\right)\left(\widehat{\mu}_{h}-\mu\right)+\left(\widehat{\mu}_{h}-\mu\right)^{2} .
\end{aligned}
$$


At this point, we integrate over $Q_{t}$. As $g$ is nonnegative, we get:

$$
\frac{1}{2} \int_{\Omega}\left|\left(\widehat{\mu}_{h}-\mu\right)(t)\right|^{2}+\int_{0}^{t}\left\|\left(\widehat{\mu}_{h}-\mu\right)(s)\right\|_{V}^{2} \mathrm{~d} s \leq \sum_{j=1}^{7} I_{j}(t),
$$

with an obvious meaning of $I_{j}(t), j=1, \ldots, 7$. Now, we estimate these integrals, but the last one. By combining the Hölder, Young, and Sobolev inequalities, and in view of (5.6), we have that

$$
\begin{aligned}
I_{1}(t) & \leq c \int_{0}^{t}\left\|\partial_{t} \widehat{\rho}_{h}(s)\right\|_{4}\left\|\left(\widehat{\mu}_{h}-\mu\right)(s)\right\|_{H}\left\|\left(\widehat{\mu}_{h}-\mu\right)(s)\right\|_{4} \mathrm{~d} s \\
& \leq c \int_{0}^{t}\left\|\partial_{t} \widehat{\rho}_{h}(s)\right\|_{V}\left\|\left(\widehat{\mu}_{h}-\mu\right)(s)\right\|_{H}\left\|\left(\widehat{\mu}_{h}-\mu\right)(s)\right\|_{V} \mathrm{~d} s \\
& \leq \sigma \int_{0}^{t}\left\|\left(\widehat{\mu}_{h}-\mu\right)(s)\right\|_{V}^{2} \mathrm{~d} s+c_{\sigma} \int_{0}^{t}\left\|\left(\widehat{\mu}_{h}-\mu\right)(s)\right\|_{H}^{2} \mathrm{~d} s,
\end{aligned}
$$

where $\sigma>0$ is arbitrary. Similarly, we infer that

$$
\begin{aligned}
I_{2}(t) & \leq 2 \int_{0}^{t}\left\|\partial_{t} \mu(s)\right\|_{H}\left\|\left(\widehat{\rho}_{h}-\rho\right)(s)\right\|_{4}\left\|\left(\widehat{\mu}_{h}-\mu\right)(s)\right\|_{4} \mathrm{~d} s \\
& \leq \sigma \int_{0}^{t}\left\|\left(\widehat{\mu}_{h}-\mu\right)(s)\right\|_{V}^{2} \mathrm{~d} s+c_{\sigma} \int_{0}^{t}\left\|\partial_{t} \mu(s)\right\|_{H}^{2}\left\|\left(\widehat{\rho}_{h}-\rho\right)(s)\right\|_{V}^{2} \mathrm{~d} s .
\end{aligned}
$$

Notice that, by means of the Gronwall lemma, we shall be able to control the last integral in terms of the $L^{1}(0, T)$-norm of the function $s \mapsto\left\|\partial_{t} \mu(s)\right\|_{H}^{2}$ (cf. (2.8)). We use a similar procedure for the next integral and notice that the same remark holds, due to (5.8). Indeed, we have that

$$
\begin{aligned}
I_{3}(t) & \leq \int_{0}^{t}\left\|\partial_{t} \widehat{\gamma}_{h}(s)\right\|_{\infty}\left\|\left(\widehat{\mu}_{h}-\mu\right)(s)\right\|_{H}\left\|\left(\bar{\mu}_{h}-\mu\right)(s)\right\|_{H} \mathrm{~d} s \\
& \leq \int_{0}^{t}\left\|\partial_{t} \widehat{\gamma}_{h}(s)\right\|_{\infty}\left\|\left(\widehat{\mu}_{h}-\mu\right)(s)\right\|_{H}\left(\left\|\left(\widehat{\mu}_{h}-\mu\right)(s)\right\|_{H}+\left\|\left(\bar{\mu}_{h}-\widehat{\mu}_{h}\right)(s)\right\|_{H}\right) \mathrm{d} s \\
& \leq \int_{0}^{t}\left\|\partial_{t} \widehat{\gamma}_{h}(s)\right\|_{\infty}^{2}\left\|\left(\widehat{\mu}_{h}-\mu\right)(s)\right\|_{H}^{2} \mathrm{~d} s+c \int_{0}^{t}\left(\left\|\left(\widehat{\mu}_{h}-\mu\right)(s)\right\|_{H}^{2}+\left\|\left(\bar{\mu}_{h}-\widehat{\mu}_{h}\right)(s)\right\|_{H}^{2}\right) \mathrm{d} s \\
& \leq c \int_{0}^{t}\left(\left\|\partial_{t} \widehat{\gamma}_{h}(s)\right\|_{\infty}^{2}+1\right)\left\|\left(\widehat{\mu}_{h}-\mu\right)(s)\right\|_{H}^{2} \mathrm{~d} s+c h^{2},
\end{aligned}
$$

where the last inequality is due to (4.32). In order to treat $I_{4}(t)$, we prove a preliminary estimate, namely, that

$$
\left|\partial_{t}\left(\widehat{\gamma}_{h}-g(\rho)\right)\right| \leq c\left\{\left|\bar{\rho}_{h}-\widehat{\rho}_{h}\right|+\left|\underline{\rho}_{h}-\widehat{\rho}_{h}\right|+\left|\widehat{\rho}_{h}-\rho\right|\right\}\left|\partial_{t} \widehat{\rho}_{h}\right|+c\left|\partial_{t}\left(\widehat{\rho}_{h}-\rho\right)\right|
$$

a.e. in $Q$. As we argue pointwise, we fix $(x, t)$ a.e. in $Q$ and choose $n$ such that $t$ belongs to the interval $(n h,(n+1) h]$; in order to simplify the notation, we omit writing at what point $(x, t)$ we work. By the mean value theorem, we find $r$ between $\rho_{n}$ and $\rho_{n+1}$ such that

$$
\begin{aligned}
\partial_{t}\left(\widehat{\gamma}_{h}-g(\rho)\right) & =\frac{g\left(\rho_{n+1}\right)-g\left(\rho_{n}\right)}{h}-g^{\prime}(\rho) \partial_{t} \rho=g^{\prime}(r) \frac{\rho_{n+1}-\rho_{n}}{h}-g^{\prime}(\rho) \partial_{t} \rho \\
& =g^{\prime}(r) \partial_{t} \widehat{\rho}_{h}-g^{\prime}(\rho) \partial_{t} \rho=\left(g^{\prime}(r)-g^{\prime}(\rho)\right) \partial_{t} \widehat{\rho}_{h}+g^{\prime}(\rho)\left(\partial_{t} \widehat{\rho}_{h}-\partial_{t} \rho\right) .
\end{aligned}
$$


As $g^{\prime}$ is bounded and Lipschitz continuous, we infer that

$$
\left|\partial_{t}\left(\widehat{\gamma}_{h}-g(\rho)\right)\right| \leq c|r-\rho|\left|\partial_{t} \widehat{\rho}_{h}\right|+c\left|\partial_{t} \widehat{\rho}_{h}-\partial_{t} \rho\right| .
$$

On the other hand, we have

$|r-\rho| \leq\left|r-\rho_{n}\right|+\left|\rho_{n}-\rho\right| \leq\left|\rho_{n+1}-\rho_{n}\right|+\left|\rho_{n}-\rho\right|=\left|\bar{\rho}_{h}-\underline{\rho}_{h}\right|+\left|\underline{\rho}_{h}-\rho\right| \leq\left|\bar{\rho}_{h}-\widehat{\rho}_{h}\right|+2\left|\underline{\rho}_{h}-\widehat{\rho}_{h}\right|+\left|\widehat{\rho}_{h}-\rho\right|$.

Hence, (5.15) follows, and we can use it to estimate $I_{4}(t)$. We also account for the boundedness of $\mu$ and for identity (2.24) and the analogue identity concerning $\underline{z}_{h}$. We have:

$$
\begin{aligned}
I_{4}(t) \leq & c \int_{0}^{t} \int_{\Omega}\left\{\left|\bar{\rho}_{h}-\widehat{\rho}_{h}\right|+\left|\underline{\rho}_{h}-\widehat{\rho}_{h}\right|+\left|\widehat{\rho}_{h}-\rho\right|\right\}\left|\partial_{t} \widehat{\rho}_{h}\right|\left|\widehat{\mu}_{h}-\mu\right| \\
& +c \int_{0}^{t} \int_{\Omega}\left|\partial_{t}\left(\widehat{\rho}_{h}-\rho\right)\right|\left|\widehat{\mu}_{h}-\mu\right| \\
\leq & c \int_{0}^{t}\left\{\left\|\bar{\rho}_{h}(s)-\widehat{\rho}_{h}(s)\right\|_{H}^{2}+\left\|\underline{\rho}_{h}(s)-\widehat{\rho}_{h}(s)\right\|_{H}^{2}+\left\|\widehat{\rho}_{h}(s)-\rho(s)\right\|_{H}^{2}\right\}\left\|\partial_{t} \widehat{\rho}_{h}(s)\right\|_{\infty}^{2} \mathrm{~d} s \\
& +\frac{1}{2} \int_{0}^{t}\left\|\partial_{t}\left(\widehat{\rho}_{h}-\rho\right)(s)\right\|_{H}^{2} \mathrm{~d} s+c \int_{0}^{t}\left\|\left(\widehat{\mu}_{h}-\mu\right)(s)\right\|_{H}^{2} \mathrm{~d} s \\
\leq & c h^{2}\left\|\partial_{t} \widehat{\rho}_{h}\right\|_{L^{\infty}(0, T ; H)}^{2}\left\|\partial_{t} \widehat{\rho}_{h}\right\|_{L^{2}\left(0, T ; L^{\infty}(\Omega)\right)}^{2}+c \int_{0}^{t}\left\|\partial_{t} \widehat{\rho}_{h}(s)\right\|_{\infty}^{2}\left\|\widehat{\rho}_{h}(s)-\rho(s)\right\|_{H}^{2} \mathrm{~d} s \\
& +\frac{1}{2} \int_{0}^{t}\left\|\partial_{t}\left(\widehat{\rho}_{h}-\rho\right)(s)\right\|_{H}^{2} \mathrm{~d} s+c \int_{0}^{t}\left\|\left(\widehat{\mu}_{h}-\mu\right)(s)\right\|_{H}^{2} \mathrm{~d} s ;
\end{aligned}
$$

furthermore, estimates (4.31) and (5.7) allow us to infer that

$$
\begin{aligned}
I_{4}(t) \leq & c h^{2}+c \int_{0}^{t}\left\|\partial_{t} \widehat{\rho}_{h}(s)\right\|_{\infty}^{2}\left\|\widehat{\rho}_{h}(s)-\rho(s)\right\|_{V}^{2} \mathrm{~d} s+\frac{1}{2} \int_{0}^{t}\left\|\partial_{t}\left(\widehat{\rho}_{h}-\rho\right)(s)\right\|_{H}^{2} \mathrm{~d} s \\
& +c \int_{0}^{t}\left(1+\left\|\partial_{t} \widehat{\rho}_{h}(s)\right\|_{\infty}^{2}\right)\left\|\left(\widehat{\mu}_{h}-\mu\right)(s)\right\|_{H}^{2} \mathrm{~d} s .
\end{aligned}
$$

Next, we deal with $I_{5}(t)$. By accounting for $(2.25)$ and (5.7), we deduce that

$$
\begin{aligned}
I_{5}(t) & \leq c \int_{0}^{t}\left\|\left(\widehat{\rho}_{h}-\underline{\rho}_{h}\right)(s)\right\|_{\infty}\left\|\partial_{t} \widehat{\mu}_{h}(s)\right\|_{H}\left\|\left(\widehat{\mu}_{h}-\mu\right)(s)\right\|_{H} \mathrm{~d} s \\
& \leq c h^{2}\left\|\partial_{t} \widehat{\rho}_{h}\right\|_{L^{2}\left(0, T ; L^{\infty}(\Omega)\right)}^{2}+c \int_{0}^{t}\left\|\partial_{t} \widehat{\mu}_{h}(s)\right\|_{H}^{2}\left\|\left(\widehat{\mu}_{h}-\mu\right)(s)\right\|_{H}^{2} \mathrm{~d} s \\
& \leq c h^{2}+c \int_{0}^{t}\left\|\partial_{t} \widehat{\mu}_{h}(s)\right\|_{H}^{2}\left\|\left(\widehat{\mu}_{h}-\mu\right)(s)\right\|_{H}^{2} \mathrm{~d} s .
\end{aligned}
$$

We note at once that we shall be able to control even the last terms of the last two estimates with the help of the Gronwall lemma, in view of (5.7) and (4.31), respectively. Finally, thanks to (2.25) once more, we have:

$$
\begin{aligned}
I_{6}(t) & =\int_{0}^{t} \int_{\Omega} \nabla\left(\widehat{\mu}_{h}-\bar{\mu}_{h}\right) \cdot \nabla\left(\widehat{\mu}_{h}-\mu\right) \\
& \leq \sigma \int_{0}^{t} \int_{\Omega}\left|\nabla\left(\widehat{\mu}_{h}-\mu\right)\right|^{2}+c_{\sigma} \int_{0}^{t} \int_{\Omega}\left|\nabla\left(\widehat{\mu}_{h}-\bar{\mu}_{h}\right)\right|^{2} \\
& \leq \sigma \int_{0}^{t} \int_{\Omega}\left|\nabla\left(\widehat{\mu}_{h}-\mu\right)\right|^{2}+c_{\sigma} h^{2}\left\|\nabla \partial_{t} \widehat{\mu}_{h}\right\|_{L^{2}(0, T ; H)}^{2} \\
& \leq \sigma \int_{0}^{t} \int_{\Omega}\left|\nabla\left(\widehat{\mu}_{h}-\mu\right)\right|^{2}+c_{\sigma} h,
\end{aligned}
$$


where the last inequality is a consequence of the non-sharp estimate (4.28). We stress that $I_{6}$ is the only term of order $h$ instead of $h^{2}$. At this point, we collect all the estimates of the integrals $I_{j}$ we have obtained, and come back to (5.13)-(5.14). If we choose $\sigma$ small enough, we conclude that

$$
\begin{aligned}
& \frac{1}{2}\left\|\left(\widehat{\mu}_{h}-\mu\right)(t)\right\|_{H}^{2}+\frac{1}{2} \int_{0}^{t}\left\|\left(\widehat{\mu}_{h}-\mu\right)(s)\right\|_{V}^{2} \mathrm{~d} s \\
& \leq c\left\{h+\int_{0}^{t}\left(1+\left\|\partial_{t} \widehat{\gamma}_{h}(s)\right\|_{\infty}^{2}+\left\|\partial_{t} \widehat{\mu}_{h}(s)\right\|_{H}^{2}\right)\left\|\left(\widehat{\mu}_{h}-\mu\right)(s)\right\|_{H}^{2} \mathrm{~d} s\right. \\
& \quad+\int_{0}^{t}\left(\left\|\partial_{t} \mu(s)\right\|_{H}^{2}+\left\|\partial_{t} \widehat{\rho}_{h}(s)\right\|_{\infty}^{2}\right)\left\|\widehat{\rho}_{h}(s)-\rho(s)\right\|_{V}^{2} \mathrm{~d} s \\
& \left.\quad+\int_{0}^{t} \int_{\Omega}\left|\partial_{t}\left(\widehat{\rho}_{h}-\rho\right)\right|^{2}\right\}+\frac{1}{2} \int_{0}^{t}\left\|\partial_{t}\left(\widehat{\rho}_{h}-\rho\right)(s)\right\|_{H}^{2} \mathrm{~d} s
\end{aligned}
$$

for every $t \in[0, T]$. Now, we revert to (5.11) and add it to (5.17). After rearranging, we apply the Gronwall lemma and obtain (2.30). This concludes the proof.

\section{Proof of Theorem 2.6}

As is clear from the proof of Theorem 2.5, to obtain estimate (2.32) there is just one step to modify, namely, the estimate of $I_{6}$ (see (5.16)), which was based on the non-sharp inequality (4.28). Thus, we only have to prove that our further assumption (2.31) implies that $I_{6}$ must be of order $h^{2}$, not $h$. Moreover, it is clear that this is true whenever we improve (4.28) and replace it by

$$
\left\|\nabla \partial_{t} \widehat{\mu}_{h}\right\|_{L^{2}(0, T ; H)}^{2} \leq c, \quad \text { i.e., } \quad h \sum_{n=0}^{N-1}\left\|\nabla \delta_{h} \mu_{n}\right\|_{H}^{2} \leq c .
$$

Hence, it suffices to prove (6.1). In order to make our argument transparent, we prove some additional estimates, the first of which holds under assumption (2.31).

Further a priori estimates. We prepare an estimate of $\left\|\delta_{h} \mu_{0}\right\|_{H}$. In view of (2.31), we write equation (2.27), with $n=0$, in the form:

$$
\left(1+2 \gamma_{0}\right) \delta_{h} \mu_{0}-h \Delta \delta_{h} \mu_{0}=\Delta \mu_{0}-\mu_{1} \delta_{h} \gamma_{0},
$$

and test it by $\delta_{h} \mu_{0}$. As $\gamma_{0}$ is nonnegative, we immediately arrive at

$$
\int_{\Omega}\left|\delta_{h} \mu_{0}\right|^{2}+h \int_{\Omega}\left|\nabla \delta_{h} \mu_{0}\right|^{2} \leq\left(\left\|\Delta \mu_{0}\right\|_{H}+\left\|\mu_{1}\right\|_{4}\left\|\delta_{h} \rho_{0}\right\|_{4}\right)\left\|\delta_{h} \mu_{0}\right\|_{H} .
$$

Thanks to (2.31), the Sobolev inequality, (4.26), and (5.5), we deduce that

$$
\left\|\delta_{h} \mu_{0}\right\|_{H}+h \int_{\Omega}\left|\nabla \delta_{h} \mu_{0}\right|^{2} \leq c
$$

Let us come to the basic estimate we need. We improve (4.21) and obtain a bound for the second-difference quotients $\delta_{h}^{2} \rho_{n}$ (see $(2.16)$ ). We write $(2.28)$, with $(n+1)$ in place of $n$, and test the difference between the 
resulting relation and (2.28) itself by $\left(\delta_{h} \rho_{n+1}-\delta_{h} \rho_{n}\right)$. We find:

$$
\begin{aligned}
& \int_{\Omega}\left|\delta_{h} \rho_{n+1}-\delta_{h} \rho_{n}\right|^{2}+h \int_{\Omega} \nabla \delta_{h} \rho_{n+1} \cdot \nabla\left(\delta_{h} \rho_{n+1}-\delta_{h} \rho_{n}\right) \\
& =-\int_{\Omega}\left(f^{\prime}\left(\rho_{n+2}\right)-f^{\prime}\left(\rho_{n+1}\right)\right)\left(\delta_{h} \rho_{n+1}-\delta_{h} \rho_{n}\right) \\
& \quad+h \int_{\Omega}\left(g^{\prime}\left(\rho_{n+1}\right) \delta_{h} \mu_{n}+\mu_{n} \delta_{h}\left(g^{\prime}\left(\rho_{n}\right)\right)\right)\left(\delta_{h} \rho_{n+1}-\delta_{h} \rho_{n}\right) \\
& \leq C h \int_{\Omega}\left(\left|\delta_{h} \rho_{n+1}\right|+\left|\delta_{h} \mu_{n}\right|+\left|\mu_{n}\right|\left|\delta_{h} \rho_{n}\right|\right)\left|\delta_{h} \rho_{n+1}-\delta_{h} \rho_{n}\right| .
\end{aligned}
$$

By the elementary identity (2.36), we have:

$$
\nabla \delta_{h} \rho_{n+1} \cdot \nabla\left(\delta_{h} \rho_{n+1}-\delta_{h} \rho_{n}\right)=\frac{1}{2}\left|\nabla \delta_{h} \rho_{n+1}\right|^{2}-\frac{1}{2}\left|\nabla \delta_{h} \rho_{n}\right|^{2}+\frac{1}{2}\left|\nabla \delta_{h} \rho_{n+1}-\nabla \delta_{h} \rho_{n}\right|^{2} .
$$

On the other hand, by the Sobolev inequality, (4.26), and (5.5), we infer that

$$
\begin{aligned}
& C h \int_{\Omega}\left(\left|\delta_{h} \rho_{n+1}\right|+\left|\delta_{h} \mu_{n}\right|+\left|\mu_{n}\right|\left|\delta_{h} \rho_{n}\right|\right)\left|\delta_{h} \rho_{n+1}-\delta_{h} \rho_{n}\right| \\
& \leq \frac{1}{2} \int_{\Omega}\left|\delta_{h} \rho_{n+1}-\delta_{h} \rho_{n}\right|^{2}+c h^{2}\left(\left\|\delta_{h} \rho_{n+1}\right\|_{H}^{2}+\left\|\delta_{h} \mu_{n}\right\|_{H}^{2}+\left\|\mu_{n}\right\|_{4}^{2}\left\|\delta_{h} \rho_{n}\right\|_{4}^{2}\right) \\
& \leq \frac{1}{2} \int_{\Omega}\left|\delta_{h} \rho_{n+1}-\delta_{h} \rho_{n}\right|^{2}+c h^{2}\left(\left\|\delta_{h} \rho_{n+1}\right\|_{H}^{2}+\left\|\delta_{h} \mu_{n}\right\|_{H}^{2}+\left\|\mu_{n}\right\|_{V}^{2}\left\|\delta_{h} \rho_{n}\right\|_{V}^{2}\right) \\
& \leq \frac{1}{2} \int_{\Omega}\left|\delta_{h} \rho_{n+1}-\delta_{h} \rho_{n}\right|^{2}+c h^{2}\left(\left\|\delta_{h} \rho_{n+1}\right\|_{H}^{2}+\left\|\delta_{h} \mu_{n}\right\|_{H}^{2}+1\right) .
\end{aligned}
$$

Now, we combine this estimate, the identity just above, and (6.3). Then, we divide by $h$ and sum over $n=$ $0, \ldots, m-1$, where $1 \leq m \leq N-1$. We obtain:

$$
\begin{aligned}
& \frac{h}{2} \sum_{n=0}^{m-1}\left\|\delta_{h}^{2} \rho_{n}\right\|_{H}^{2}+\frac{1}{2}\left\|\nabla \delta_{h} \rho_{m}\right\|_{H}^{2}+\frac{1}{2} \sum_{n=0}^{m-1}\left\|\nabla \delta_{h} \rho_{n+1}-\nabla \delta_{h} \rho_{n}\right\|_{H}^{2} \\
& \leq \frac{1}{2}\left\|\nabla \delta_{h} \rho_{0}\right\|_{H}^{2}+c h \sum_{n=0}^{m-1}\left\|\delta_{h} \rho_{n+1}\right\|_{H}^{2}+c h \sum_{n=0}^{m-1}\left\|\delta_{h} \mu_{n}\right\|_{H}^{2}+c .
\end{aligned}
$$

At this point, by (5.5), (4.6), and (4.26), we conclude that

$$
h \sum_{n=0}^{N-2}\left\|\delta_{h}^{2} \rho_{n}\right\|_{H}^{2} \leq c .
$$

Consequence. With a view toward deriving an estimate for $\delta_{h}^{2} \gamma_{n}$, we begin by arguing pointwise. So, for a.a. $(x, t) \in Q$ (once again we omit writing at what point of $Q$ we work) and for suitable $r_{1}$ between $\rho_{n+2}$ and $\rho_{n+1}$, and $r_{2}$ between $\rho_{n}$ and $\rho_{n+1}$, we have by the Taylor formula:

$$
\begin{aligned}
\left|\delta_{h}^{2} \gamma_{n}\right|= & h^{-2}\left|g\left(\rho_{n+2}\right)-g\left(\rho_{n+1}\right)+g\left(\rho_{n}\right)-g\left(\rho_{n+1}\right)\right| \\
= & h^{-2} \mid g^{\prime}\left(\rho_{n+1}\right)\left(\rho_{n+2}-\rho_{n+1}\right)+\frac{1}{2} g^{\prime \prime}\left(r_{1}\right)\left(\rho_{n+2}-\rho_{n+1}\right)^{2} \\
& \quad+g^{\prime}\left(\rho_{n+1}\right)\left(\rho_{n}-\rho_{n+1}\right)+\frac{1}{2} g^{\prime \prime}\left(r_{2}\right)\left(\rho_{n}-\rho_{n+1}\right)^{2} \mid \\
\leq & c\left|\delta_{h}^{2} \rho_{n}\right|+c\left(\left|\delta_{h} \rho_{n+1}\right|^{2}+\left|\delta_{h} \rho_{n}\right|^{2}\right) .
\end{aligned}
$$


Now, we square this pointwise estimate, integrate over $\Omega$, sum over $n$, and deduce that

$$
h \sum_{n=0}^{N-2}\left\|\delta_{h}^{2} \gamma_{n}\right\|_{H}^{2} \leq c h \sum_{n=0}^{N-2}\left\|\delta_{h}^{2} \rho_{n}\right\|_{H}^{2}+c h \sum_{n=0}^{N-1}\left\|\delta_{h} \rho_{n}\right\|_{4}^{4} .
$$

Then, (6.4), the Sobolev inequality, and (5.5) yield:

$$
h \sum_{n=0}^{N-2}\left\|\delta_{h}^{2} \gamma_{n}\right\|_{H}^{2} \leq c
$$

Proof of Theorem 2.6. As said before, it suffices to prove (6.1). We reason that, in order to obtain the analogous estimate for the solution to the continuous problem, one first differentiates (2.12) with respect to time and then tests the resulting equality by $\partial_{t} \mu$; this yields the desired term $\int_{0}^{t} \int_{\Omega}\left|\nabla \partial_{t} \mu\right|^{2}$ on the left-hand side. The idea is to perform the corresponding procedure on the discrete equation (2.27). However, it turns out that the calculation in the discrete case becomes simpler if one tests by the analogue of the product $(1+2 g(\rho)) \partial_{t} \mu$. To simplify the notation, we introduce the vector $\pi$ defined by

$$
\pi_{n}:=\left(1+2 \gamma_{n}\right) \delta_{h} \mu_{n} \quad \text { for } n=0, \ldots, N-1
$$

We write $(2.27)$ with $(n+1)$ in place of $n$, and take the difference between the resulting equality and $(2.27)$ itself. Then, we test this difference by $\pi_{n+1}$ and integrate over $\Omega$. By taking the elementary identity (2.36) into account, we obtain for $n=0, \ldots, N-2$ that

$$
\begin{aligned}
& \frac{1}{2} \int_{\Omega}\left|\pi_{n+1}\right|^{2}-\frac{1}{2} \int_{\Omega}\left|\pi_{n}\right|^{2}+\frac{1}{2} \int_{\Omega}\left|\pi_{n+1}-\pi_{n}\right|^{2}+h \int_{\Omega} \nabla \delta_{h} \mu_{n+1} \cdot \nabla \pi_{n+1} \\
& =-\int_{\Omega}\left(\mu_{n+2} \delta_{h} \gamma_{n+1}-\mu_{n+1} \delta_{h} \gamma_{n}\right) \pi_{n+1} .
\end{aligned}
$$

By computing the fourth term on the left-hand side with the help of (6.6), recalling that $g$ is nonnegative, and rearranging, we deduce that

$$
\begin{aligned}
& \int_{\Omega}\left|\pi_{n+1}\right|^{2}-\int_{\Omega}\left|\pi_{n}\right|^{2}+\int_{\Omega}\left|\pi_{n+1}-\pi_{n}\right|^{2}+2 h \int_{\Omega}\left|\nabla \delta_{h} \mu_{n+1}\right|^{2} \\
& \leq-2 \int_{\Omega}\left(\mu_{n+2} \delta_{h} \gamma_{n+1}-\mu_{n+1} \delta_{h} \gamma_{n}\right) \pi_{n+1}-4 h \int_{\Omega} \delta_{h} \mu_{n+1} \nabla \delta_{h} \mu_{n+1} \cdot \nabla \gamma_{n+1} \\
& =-2 \int_{\Omega} \pi_{n+1}\left(\mu_{n+2}-\mu_{n+1}\right) \delta_{h} \gamma_{n+1}-2 \int_{\Omega} \pi_{n+1} \mu_{n+1}\left(\delta_{h} \gamma_{n+1}-\delta_{h} \gamma_{n}\right) \\
& \quad-4 h \int_{\Omega} \delta_{h} \mu_{n+1} \nabla \delta_{h} \mu_{n+1} \cdot \nabla \gamma_{n+1} \\
& =-2 h \int_{\Omega} \pi_{n+1} \delta_{h} \mu_{n+1} \delta_{h} \gamma_{n+1}-2 h \int_{\Omega} \pi_{n+1} \mu_{n+1} \delta_{h}^{2} \gamma_{n} \\
& \quad-4 h \int_{\Omega} \delta_{h} \mu_{n+1} \nabla \delta_{h} \mu_{n+1} \cdot \nabla \gamma_{n+1} .
\end{aligned}
$$


Now, we estimate each term of the right-hand side separately, before summing over $n$, in order to simplify the notation. For the first one, we use Hölder and Sobolev inequalities, and estimates (5.5) and (6.4). We have:

$$
\begin{aligned}
-2 h \int_{\Omega} \pi_{n+1} \delta_{h} \mu_{n+1} \delta_{h} \gamma_{n+1} & \leq 2 h\left\|\pi_{n+1}\right\|_{4}\left\|\delta_{h} \mu_{n+1}\right\|_{H}\left\|\delta_{h} \gamma_{n+1}\right\|_{4} \leq c h\left\|\delta_{h} \mu_{n+1}\right\|_{V}\left\|\delta_{h} \mu_{n+1}\right\|_{H}\left\|\delta_{h} \rho_{n+1}\right\|_{V} \\
& \leq \frac{h}{4}\left(\left\|\nabla \delta_{h} \mu_{n+1}\right\|_{H}^{2}+\left\|\delta_{h} \mu_{n+1}\right\|_{H}^{2}\right)+c h\left\|\delta_{h} \mu_{n+1}\right\|_{H}^{2} \\
& \leq \frac{h}{4}\left\|\nabla \delta_{h} \mu_{n+1}\right\|_{H}^{2}+c h\left\|\pi_{n+1}\right\|_{H}^{2} \\
& \leq \frac{h}{4}\left\|\nabla \delta_{h} \mu_{n+1}\right\|_{H}^{2}+c h\left\|\pi_{n}\right\|_{H}^{2}+C h\left\|\pi_{n+1}-\pi_{n}\right\|_{H}^{2} .
\end{aligned}
$$

For $h$ small enough, namely, for $h \leq 1 /(3 C)$, we conclude that

$$
-2 h \int_{\Omega} \pi_{n+1} \delta_{h} \mu_{n+1} \delta_{h} \gamma_{n+1} \leq \frac{h}{4}\left\|\nabla \delta_{h} \mu_{n+1}\right\|_{H}^{2}+c h\left\|\pi_{n}\right\|_{H}^{2}+\frac{1}{3}\left\|\pi_{n+1}-\pi_{n}\right\|_{H}^{2} .
$$

Next, by (4.26), we similarly have:

$$
\begin{aligned}
& -2 h \int_{\Omega} \pi_{n+1} \mu_{n+1} \delta_{h}^{2} \gamma_{n} \leq c h\left\|\delta_{h} \mu_{n+1}\right\|_{4}\left\|\mu_{n+1}\right\|_{4}\left\|\delta_{h}^{2} \gamma_{n}\right\|_{H} \\
& \leq \frac{h}{4}\left(\left\|\nabla \delta_{h} \mu_{n+1}\right\|_{H}^{2}+\left\|\delta_{h} \mu_{n+1}\right\|_{H}^{2}\right)+c h\left\|\mu_{n+1}\right\|_{V}^{2}\left\|\delta_{h}^{2} \gamma_{n}\right\|_{H}^{2} \\
& \leq \frac{h}{4}\left\|\nabla \delta_{h} \mu_{n+1}\right\|_{H}^{2}+h\left\|\pi_{n}\right\|_{H}^{2}+\frac{1}{3}\left\|\pi_{n+1}-\pi_{n}\right\|_{H}^{2}+c h\left\|\delta_{h}^{2} \gamma_{n}\right\|_{H}^{2},
\end{aligned}
$$

for sufficiently small $h$. Finally, by accounting for (4.23), Sobolev inequality, and the compactness inequality (2.35), we have that, for $h$ small enough,

$$
\begin{aligned}
& -4 h \int_{\Omega} \delta_{h} \mu_{n+1} \nabla \delta_{h} \mu_{n+1} \cdot \nabla \gamma_{n+1} \leq c h\left\|\delta_{h} \mu_{n+1}\right\|_{4}\left\|\nabla \delta_{h} \mu_{n+1}\right\|_{H}\left\|\nabla \rho_{n+1}\right\|_{4} \\
& \leq \frac{h}{4}\left\|\nabla \delta_{h} \mu_{n+1}\right\|_{H}^{2}+c h\left\|\delta_{h} \mu_{n+1}\right\|_{4}^{2}\left\|\nabla \rho_{n+1}\right\|_{V}^{2} \leq \frac{h}{4}\left\|\nabla \delta_{h} \mu_{n+1}\right\|_{H}^{2}+c h\left\|\delta_{h} \mu_{n+1}\right\|_{4}^{2} \\
& \leq \frac{h}{4}\left\|\nabla \delta_{h} \mu_{n+1}\right\|_{H}^{2}+h\left(\frac{1}{4}\left\|\nabla \delta_{h} \mu_{n+1}\right\|_{H}^{2}+c\left\|\delta_{h} \mu_{n+1}\right\|_{H}^{2}\right) \\
& \leq \frac{h}{2}\left\|\nabla \delta_{h} \mu_{n+1}\right\|_{H}^{2}+c h\left\|\pi_{n+1}\right\|_{H}^{2} \leq \frac{h}{2}\left\|\nabla \delta_{h} \mu_{n+1}\right\|_{H}^{2}+c h\left\|\pi_{n}\right\|_{H}^{2}+\frac{1}{3}\left\|\pi_{n+1}-\pi_{n}\right\|_{H}^{2} .
\end{aligned}
$$

At this point, we combine the inequalities just obtained with (6.7) and note that the terms involving $\pi_{n+1}-\pi_{n}$ cancel out. Then, we sum over $n=0, \ldots, m-1$, with $1 \leq m \leq(N-1)$. We obtain:

$$
\left\|\pi_{m}\right\|_{H}^{2}+h \sum_{n=0}^{m-1}\left\|\nabla \delta_{h} \mu_{n+1}\right\|_{H}^{2} \leq\left\|\pi_{0}\right\|_{H}^{2}+h \sum_{n=0}^{m-1}\left\|\pi_{n}\right\|_{H}^{2}+h \sum_{n=0}^{m-1}\left\|\delta_{h}^{2} \gamma_{n}\right\|_{H}^{2}
$$

and the discrete Gronwall lemma allows us to deduce that

$$
\left\|\pi_{m}\right\|_{H}^{2}+h \sum_{n=0}^{m-1}\left\|\nabla \delta_{h} \mu_{n+1}\right\|_{H}^{2} \leq c\left(\left\|\pi_{0}\right\|_{H}^{2}+c h \sum_{n=0}^{N-2}\left\|\delta_{h}^{2} \gamma_{n}\right\|_{H}^{2}\right)
$$

for $1 \leq m \leq(N-1)$. From (6.2) and (6.5), we infer that

$$
h \sum_{n=0}^{N-2}\left\|\nabla \delta_{h} \mu_{n+1}\right\|_{H}^{2} \leq c .
$$

This and (6.2) yield (6.1), and the proof is complete. 
Remark 6.1. As a consequence of estimates (6.1) and (6.4), the solution to the continuous problem enjoys the following additional regularity properties:

$$
\nabla \partial_{t} \mu \in L^{2}(Q) \quad \text { and } \quad \partial_{t}^{2} \rho \in L^{2}(Q) .
$$

This can give even more: for instance, equation (2.13) can be differentiated with respect to time, to show that $\Delta \partial_{t} \rho$ belongs to $L^{2}(Q)$ as well, so as to conclude that

$$
\rho \in H^{2}(0, T ; H) \cap H^{1}(0, T ; W) .
$$

However, this regularity result could be proved formally and directly for the continuous problem.

Acknowledgements. The present paper benefits from the support of the MIUR-PRIN Grant 2010A2TFX2 "Calculus of variations" for PC and GG, the GAČR Grant P201/10/2315 and RVO: 67985840 for PK, the DFG Research Center MATHEON in Berlin for JS.

\section{REFERENCES}

[1] F. Bai, C.M. Elliott, A. Gardiner, A. Spence and A.M. Stuart, The viscous Cahn-Hilliard equation. I. Computations. Nonlinearity 8 (1995) 131-160.

[2] J.W. Barrett and J.F. Blowey, An error bound for the finite element approximation of the Cahn-Hilliard equation with logarithmic free energy. Numer. Math. 72 (1995) 1-20.

[3] J.W. Barrett and J.F. Blowey, An improved error bound for a finite element approximation of a model for phase separation of a multi-component alloy with a concentration dependent mobility matrix. Numer. Math. 88 (2001) 255-297.

[4] J.W. Barrett, J.F. Blowey and H. Garcke, Finite element approximation of the Cahn-Hilliard equation with degenerate mobility. SIAM J. Numer. Anal. 37 (1999) 286-318.

[5] J.W. Barrett, J.F. Blowey and H. Garcke, On fully practical finite element approximations of degenerate Cahn-Hilliard systems. ESAIM: M2AN 35 (2001) 713-748.

[6] S. Bartels and R. Müller, A posteriori error controlled local resolution of evolving interfaces for generalized Cahn-Hilliard equations. Interfaces Free Bound. 12 (2010) 45-73.

[7] S. Bartels and R. Müller, Error control for the approximation of Allen-Cahn and Cahn-Hilliard equations with a logarithmic potential. Numer. Math. 119 (2011) 409-435.

[8] J.F. Blowey and C.M. Elliott, The Cahn-Hilliard gradient theory for phase separation with nonsmooth free energy. II. Numerical analysis. Eur. J. Appl. Math. 3 (1992) 147-179.

[9] E. Bonetti, Global solvability of a dissipative Frémond model for shape memory alloys. II. Existence. Quart. Appl. Math. 62 (2004) 53-76.

[10] H. Brézis, Opérateurs maximaux monotones et semi-groupes de contractions dans les espaces de Hilbert. North-Holland Mathematics Studies, no. 5. Notas de Matemática. North-Holland Publishing Co., Amsterdam (1973).

[11] C. Carstensen and P. Plecháč, Numerical analysis of a relaxed variational model of hysteresis in two-phase solids. ESAIM: M2AN 35 (2001) 865-878.

[12] Z. Chen, R.H. Nochetto and A. Schmidt, Error control and adaptivity for a phase relaxation model. ESAIM: M2AN 34 (2000) $775-797$.

[13] L. Cherfils, M. Petcu and M. Pierre, A numerical analysis of the Cahn-Hilliard equation with dynamic boundary conditions. Discrete Contin. Dyn. Syst. 27 (2010) 1511-1533.

[14] E. Chiodaroli, A dissipative model for hydrogen storage: existence and regularity results. Math. Methods Appl. Sci. 34 (2011) 642-669.

[15] P. Colli, M. Frémond and O. Klein, Global existence of a solution to a phase field model for supercooling. Nonlinear Anal. Real World Appl. 2 (2001) 523-539.

[16] P. Colli, G. Gilardi, P. Podio-Guidugli and J. Sprekels, Well-posedness and long-time behavior for a nonstandard viscous Cahn-Hilliard system. SIAM J. Appl. Math. 71 (2011) 1849-1870.

[17] P. Colli, G. Gilardi, P. Podio-Guidugli and J. Sprekels, Global existence and uniqueness for a singular/degenerate Cahn-Hilliard system with viscosity. J. Differ. Equ. 254 (2013) 4217-4244.

[18] C. Eck, B. Jadamba and P. Knabner, Error estimates for a finite element discretization of a phase field model for mixtures. SIAM J. Numer. Anal. 47 (2010) 4429-4445.

[19] S. Frigeri, P. Krejčí and U. Stefanelli, Quasistatic isothermal evolution of shape memory alloys. Math. Models Methods Appl. Sci. 21 (2011) 2409-2432.

[20] G. Gilardi and U. Stefanelli, Time-discretization and global solution for a doubly nonlinear Volterra equation. J. Differ. Equ. 228 (2006) 707-736.

[21] C. Gräser and R. Kornhuber, Multigrid methods for obstacle problems. J. Comput. Math. 27 (2009) 1-44. 
[22] C. Gräser and R. Kornhuber, Nonsmooth Newton methods for set-valued saddle point problems. SIAM J. Numer. Anal. 47 (2009) 1251-1273.

[23] C. Gräser, R. Kornhuber and U. Sack, Nonsmooth Schur-Newton methods for vector-valued Cahn-Hilliard equations. Freie Universität Berlin, Fachbereich Mathematik und Informatik, Serie A Preprint no. 01 (2013) 1-16.

[24] J.W. Jerome, Approximation of nonlinear evolution systems, vol. 164 of Math. Sci. Eng. Academic Press Inc., Orlando, FL (1983).

[25] D. Kessler and J.-F. Scheid, A priori error estimates of a finite-element method for an isothermal phase-field model related to the solidification process of a binary alloy. IMA J. Numer. Anal. 22 (2002) 281-305.

[26] J. Kim, Phase-field models for multi-component fluid flows. Commun. Comput. Phys. 12 (2012) 613-661.

[27] P. Krejčí and U. Stefanelli, Well-posedness of a thermo-mechanical model for shape memory alloys under tension. ESAIM: M2AN 44 (2010) 1239-1253.

[28] A. Mielke, L. Paoli and A. Petrov, On existence and approximation for a 3D model of thermally induced phase transformations in shape-memory alloys. SIAM J. Math. Anal. 41 (2009) 1388-1414.

[29] P. Podio-Guidugli, Models of phase segregation and diffusion of atomic species on a lattice. Ric. Mat. 55 (2006) $105-118$.

[30] M. Röger, Existence of weak solutions for the Mullins-Sekerka flow. SIAM J. Math. Anal. 37 (2005) 291-301.

[31] A. Segatti, Error estimates for a variable time-step discretization of a phase transition model with hyperbolic momentum. Numer. Funct. Anal. Optim. 25 (2004) 547-569.

[32] J. Simon, Compact sets in the space $L^{p}(0, T ; B)$. Ann. Mat. Pura Appl. 146 (1987) 65-96.

[33] U. Stefanelli, Error control of a nonlinear evolution problem related to phase transitions. Numer. Funct. Anal. Optim. 20 (1999) 585-608.

[34] U. Stefanelli, Error control for a time-discretization of the full one-dimensional Frémond model for shape memory alloys. Adv. Math. Sci. Appl. 10 (2000) 917-936.

[35] U. Stefanelli, Analysis of a variable time-step discretization for a phase transition model with micro-movements. Commun. Pure Appl. Anal. 5 (2006) 657-671.

[36] C.L.D. Vaz, Rothe's method for an isothermal phase-field model of a binary alloy with convection. Mat. Contemp. 32 (2007) $221-251$. 